Excitation energies, radiative and autoionization rates, dielectronic satellite lines and dielectronic recombination rates for excited states of Ag-like W from Pd-like W

This article has been downloaded from IOPscience. Please scroll down to see the full text article. 2011 J. Phys. B: At. Mol. Opt. Phys. 44035005

(http://iopscience.iop.org/0953-4075/44/3/035005)

View the table of contents for this issue, or go to the journal homepage for more

Download details:

IP Address: 129.74.250.206

The article was downloaded on 20/01/2011 at 03:17

Please note that terms and conditions apply. 


\title{
Excitation energies, radiative and
} autoionization rates, dielectronic satellite lines and dielectronic recombination rates for excited states of Ag-like W from Pd-like W

\author{
U I Safronova ${ }^{1}$, A S Safronova ${ }^{1}$, P Beiersdorfer ${ }^{2}$ and W R Johnson ${ }^{3}$ \\ ${ }^{1}$ Physics Department, University of Nevada, Reno, NV 89557, USA \\ ${ }^{2}$ Physics Division, Lawrence Livermore National Laboratory, Livermore, CA 94550, USA \\ ${ }^{3}$ Department of Physics, University of Notre Dame, Notre Dame, IN 46556, USA \\ E-mail: ulyanas@unr.edu
}

Received 17 September 2010, in final form 3 December 2010

Published 19 January 2011

Online at stacks.iop.org/JPhysB/44/035005

\begin{abstract}
Energy levels, radiative transition probabilities and autoionization rates for $[\mathrm{Kr}] 4 \mathrm{~d}^{9} 4 \mathrm{f} n l(n=$ 4-9), $[\mathrm{Kr}] 4 \mathrm{~d}^{9} 5 l^{\prime} n l(n=5-9)$ and $\left[\mathrm{Kr}^{\prime} 4 \mathrm{~d}^{9} 6 l^{\prime} n l(n=6-7)\right.$ states in Ag-like tungsten $\left(\mathrm{W}^{27+}\right)$ are calculated using the relativistic many-body perturbation theory method, the multiconfiguration relativistic Hebrew University Lawrence Livermore Atomic Code and the

Hartree-Fock-relativistic method. Branching ratios relative to the first threshold and intensity factors are calculated for satellite lines, and dielectronic recombination (DR) rate coefficients are determined for the singly excited $[\mathrm{Kr}] 4 \mathrm{~d}^{10} n l(n=4-9)$ states. The total DR rate coefficient is derived as a function of electron temperature. These atomic data are important in the modelling of N-shell radiation spectra of heavy ions generated in various collision as well as plasma experiments. The tungsten data are particularly important for fusion application.

(Some figures in this article are in colour only in the electronic version)
\end{abstract}

\section{Introduction}

The use of tungsten as a plasma-facing material in magnetic fusion devices has increased rapidly in recent years. The reason is that tungsten is thought to be the best material to withstand the high thermal loads produced by burning plasma. For example, tungsten is the leading candidate material in the design of divertor in the ITER (Latin, 'the way') tokamak [1]. In anticipation of the needs of ITER arising from the use of tungsten, many present-day devices have included tungsten components inside the vacuum vessel. Examples are the Alcator C-Mod tokamak, the Joint European Torus and the ASDEX Upgrade tokamak [2-4]. As a result of the use of tungsten-containing plasma-facing components, tungsten ions are now indigenous constituents of modern magnetic fusion plasmas.
Line radiation from tungsten plasma impurities has been studied since the first use of tungsten in tokamaks [5-7], and these studies continue with increased intensity today [8-12]. The electron temperatures in most devices are such that tungsten attains charge states around 30 times ionized, among which silver-like and palladium-like tungsten are often prominent [13].

Atomic data are needed to interpret the observed spectra. Such data include not only spectral line positions but also dielectronic recombination (DR) data, as DR produces excited levels that can decay by photon emission, which completes the recombination process. In addition, DR data are needed for the modelling of the ionization balance in the plasma.

The DR process in tungsten ions was investigated recently in a number of publications [14-19]. Dielectronic satellite lines and DR rates for excited states of Na-like W from Ne-like 
$\mathrm{W}$ and for excited states of Mg-like $\mathrm{W}$ from Na-like $\mathrm{W}$ were presented by Safronova et al in [14] and [15], respectively. The relativistic many-body perturbation theory method (RMBPT code), the multiconfiguration relativistic Hebrew University Lawrence Livermore Atomic Code (HULLAC code) and the Hartree-Fock-relativistic method (COWAN code) were used to perform large-scale calculations of atomic parameters (excitation energies, radiative and autoionization rates) to evaluate DR rate coefficients and build synthetic satellite spectra.

$\mathrm{X}$-ray spectroscopic measurements of the DR resonances of $\mathrm{W}^{60+}-\mathrm{W}^{67+}$ ions were performed by Biedermann and Radtke in [16]. Dielectronic and radiative recombination of $\mathrm{Si}$ - to N-like tungsten ions was investigated by Biedermann et al in [17]. In this case, highly charged tungsten ions were produced, stored and excited using the Berlin electron beam ion trap (EBIT). The measurement of x-rays from $n=2-$ 3, 2-4 and higher DR resonance transitions was compared to relativistic calculations of the DR cross sections and rate coefficients calculated with the HULLAC code [17].

$A b$ initio calculation of the total DR rate coefficient from the ground state $3 s^{2} 3 p^{6} 3 d^{9}$ of Co-like tungsten was performed employing the flexible atomic code (FAC) $[18,19]$. The results show that the $3 \mathrm{p}^{6} 3 \mathrm{~d}^{8} 4 l n^{\prime} l^{\prime}$ complex gives the most important contribution $(>66 \%)$ to the total DR rate coefficient. The $3 \mathrm{p}^{5} 3 \mathrm{~d}^{10} n^{\prime} l^{\prime}, 3 \mathrm{p}^{5} 3 \mathrm{~d}^{9} 4 \ln ^{\prime} l^{\prime}$ and $3 \mathrm{p}^{6} 3 \mathrm{~d}^{8} 5 \ln ^{\prime} l^{\prime}$ complexes can also give significant contributions to the total $\mathrm{DR}$ rate at relatively high electron temperature [19].

Dalhed et al [20] underlined that their paper presented for the first time an explicit calculation of the partial DR rate coefficients via the $3 l 3 l^{\prime}$ manifold for $17 \mathrm{Ne}$-like ions ranging from $\mathrm{Ar}^{8+}$ to $\mathrm{W}^{64+}$, using relativistic multiconfiguration wavefunctions to calculate both the Auger and radiative matrix elements. Unfortunately, no numerical results were given for those atomic properties.

Relativistic calculations of the DR cross section and rate coefficient for Ne-like tungsten $\left(\mathrm{W}^{64+}\right)$ in the ground state $2 \mathrm{~s}^{2} 2 \mathrm{p}^{6}$ were performed by Behar et al [21]. The DR contributions of the most important Na-like doubly excited configuration complexes, namely $(2 \mathrm{~s} 2 \mathrm{p})^{7} 3 \ln l^{\prime}(n=3-13)$ and $(2 \mathrm{~s} 2 \mathrm{p})^{7} 4 l 4 l^{\prime}$, were calculated by level-by-level computations. All the basic atomic quantities used in the calculations were obtained by using the HULLAC code. The same code was used also by Peleg et al in [22] to evaluate the total DR rate coefficient for Ar-like tungsten, $\mathrm{W}^{56+}$. Ab initio calculations of the total DR rate coefficient of Ni-like tungsten $\left(\mathrm{W}^{46+}\right)$ in the ground state were performed by Behar et al [23], again using the HULLAC atomic code package. The presented level-bylevel calculations included the DR contributions of all of the levels (over 17000) in the Cu-like inner-shell excited $3 \mathrm{~d}^{9} 4 \ln l^{\prime}$ $(n \leqslant 9), 3 \mathrm{p}^{5} 3 \mathrm{~d}^{10} 4 \ln l^{\prime}(n \leqslant 5)$ and $3 \mathrm{~s}^{3} \mathrm{p}^{6} 3 \mathrm{~d}^{10} 4 \ln l^{\prime}(n \leqslant 5)$ configurations [23].

There are only few energy values for $\mathrm{W}^{27+}$ presented in the recent NIST compilation of tungsten data [24]: the ionization potential and the splitting of the ground doublet $4 \mathrm{f}^{2} \mathrm{~F}_{5 / 2,7 / 2}$ (7109 and 33.0 in units of $10^{3} \mathrm{~cm}^{-1}$, respectively).

The RMBPT values presented in [25] gave $7105 \times$ $10^{3} \mathrm{~cm}^{-1}$ for the ionization potential and $29.5 \times 10^{3} \mathrm{~cm}^{-1}$ for the $4 \mathrm{f}^{2} \mathrm{~F}_{5 / 2,7 / 2}$ ground state doublet in $\mathrm{W}^{27+}$. The value for the ionization potential agrees with the NIST value within the uncertainty of the latter $\left( \pm 13000 \mathrm{~cm}^{-1}\right.$ [24]).

The difference of the RMBPT value [25] for the 4f ${ }^{2} \mathrm{~F}_{5 / 2,7 / 2}$ ground doublet splitting $\left(29554 \mathrm{~cm}^{-1}\right)$ from the NIST recommended value $\left(33000 \mathrm{~cm}^{-1}\right)$ exceeds the uncertainty of $800 \mathrm{~cm}^{-1}$ [24]. For this doublet splitting, it was found in [25] that the dominant contribution $\left(30338 \mathrm{~cm}^{-1}\right)$ comes from the Dirac-Fock energy $E^{(\mathrm{DF})}$. The sum of the RMBPT contributions $\left(E^{(2)}=703 \mathrm{~cm}^{-1}, E^{(3)}=\right.$ $-114 \mathrm{~cm}^{-1}, B^{(1)}=-1499 \mathrm{~cm}^{-1}, B^{(2)}=126 \mathrm{~cm}^{-1}$ ) is equal to $-784 \mathrm{~cm}^{-1}$. As a result, the difference of $3500 \mathrm{~cm}^{-1}$ between our RMBPT value and the recommended NIST result [24] for the $4 \mathrm{f}^{2} \mathrm{~F}_{5 / 2,7 / 2}$ ground state doublet splitting has been puzzling [25].

Energy levels of the $4 \mathrm{~d}^{10} 4 \mathrm{f}, 4 \mathrm{~d}^{10} 5 l$ with $l=0-3$ ) configurations of Ag-like ions with $Z \leqslant 86$ were evaluated recently by Ivanova [26] using a relativistic perturbation theory with a model potential. One of the first calculations of the excitation energies and oscillator strengths for electric dipole transitions between the $4 \mathrm{~d}^{10} n l$ low-lying states in the silver isoelectronic sequence was presented by Cheng and Kim in [27]. Relativistic Hartree-Fock wavefunctions were used in that study.

Relativistic many-body calculations of excitation energies and transition rates from core-excited states in silver-like ions were recently presented in [28]. Energies of the $[\mathrm{Kr}] 4 \mathrm{~d}^{9} 4 \mathrm{f}^{2}$, $[\mathrm{Kr}] 4 \mathrm{~d}^{9} 4 \mathrm{f} 5 l$ and $[\mathrm{Kr}] 4 \mathrm{~d}^{9} 5 l 5 l^{\prime}$ states (with $l=\mathrm{s}, \mathrm{p}, \mathrm{d}, \mathrm{f}$ ) for Ag-like ions with $Z=50-100$ were evaluated to second order in RMBPT starting from a Pd-like Dirac-Fock potential $\left([\mathrm{Kr}] 4 \mathrm{~d}^{10}\right)$. Radiative transition rates and line strengths were calculated for the $4 \mathrm{~d}-4 \mathrm{f}$ and $4 \mathrm{~d}-5 l$ electric-dipole (E1) transitions in Ag-like ions with nuclear charge $Z=50-100$ [28].

These core-excited states (often called doubly excited states) in silver-like ions have been studied experimentally over the past 30 years not only for tungsten, as mentioned earlier, but also for other elements [5, 6, 13, 29-35]. Wavelengths and energy levels for $\mathrm{I}^{6+}$ through $\mathrm{La}^{10+}$ and for $\mathrm{Ce}^{11+}$ through $\mathrm{Ho}^{20+}$ as well as for $\mathrm{W}^{27+}$ were presented in $[29,30]$. Spectra of $\mathrm{Ce}^{11+}$ through $\mathrm{Ho}^{20+}$ were obtained by Sugar and Kaufman [30] with a high voltage triggered spark and photographed on the NBS $10.7 \mathrm{~m}$ grazing incidence spectrograph. The same authors then investigated the $4 d^{10} 5 s-4 d^{9} 5 s 5 p$ array of $\mathrm{I}^{6+}$ through $\mathrm{Eu}^{16+}$ [31]. The $4 \mathrm{~d}^{10} 5 \mathrm{~s}-4 \mathrm{~d}^{9} 5 \mathrm{~s} 5 \mathrm{p}$ transitions were identified by comparison with the analogous array in the $\mathrm{Cu}$ I isoelectronic sequence, then by comparison with a calculated spectrum. The latter was obtained with scaled Hartree-Fock radial energy integrals [31]. Nearly complete $4 d^{10} 5 s-4 d^{9} 5 s 5 p$ resonance transition arrays in the Ag isoelectronic sequence were observed by Kaufman et al [32] in sliding and triggered sparks for the ions $\mathrm{In}^{2+}$ through $\mathrm{Te}^{5+}$. Wavelengths and estimated relative intensities were given as well as energy levels and eigenvectors for the upper levels. Evidence of configuration interaction was indicated by the irregular behaviour of the fitted radial energy integrals for the $4 d^{9} 5 s 5 p$ states [32]. Spectra of highly ionized Er, Yb, Hf, W, $\mathrm{Pt}$ and $\mathrm{Au}$ were observed with the TEXT tokamak at the 
Table 1. Labelling of configurations for odd-parity and even-parity complexes.

\begin{tabular}{|c|c|c|c|c|c|c|c|c|c|c|c|}
\hline \multicolumn{6}{|c|}{ Even-parity states } & \multicolumn{6}{|c|}{ Odd-parity states } \\
\hline $\bar{N}$ & Configuration & $N$ & Configuration & $N$ & $\overline{\text { Configuration }}$ & $\bar{N}$ & Configuration & $N$ & Configuration & $N$ & Configuration \\
\hline 1 & $4 d^{10} 5 s$ & 38 & $4 d^{9} 5 p 5 f$ & 75 & $4 d^{9} 5 \mathrm{~d} 8 \mathrm{~s}$ & 1 & $4 d^{10} 5 p$ & 38 & $4 d^{9} 5 f 6 g$ & 75 & $4 d^{9} 5 g 9 p$ \\
\hline 2 & $4 d^{10} 5 d$ & 39 & $4 d^{9} 5 d 5 g$ & 76 & $4 d^{9} 5 d 8 d$ & 2 & $4 d^{10} 4 f$ & 39 & $4 d^{9} 5 g 6 \mathrm{p}$ & 76 & $4 d^{9} 5 g 9 f$ \\
\hline 3 & $4 d^{10} 5 g$ & 40 & $4 d^{9} 6 s 6 d$ & 77 & $4 d^{9} 5 d 8 g$ & 3 & $4 d^{10} 5 f$ & 40 & $4 d^{9} 5 g 6 f$ & 77 & $4 d^{9} 6 s 7 p$ \\
\hline 4 & $4 d^{10} 6 s$ & 41 & $4 d^{9} 6 s 6 g$ & 78 & $4 d^{9} 5 f 8 p$ & 4 & $4 d^{10} 6 p$ & 41 & $4 d^{9} 5 s 7 p$ & 78 & $4 d^{9} 6 s 7 f$ \\
\hline 5 & $4 d^{10} 6 d$ & 42 & $4 d^{9} 6 d 6 g$ & 79 & $4 d^{9} 5 f 8 f$ & 5 & $4 d^{10} 6 f$ & 42 & $4 d^{9} 5 s 7 f$ & 79 & $4 d^{9} 6 \mathrm{p} 7 \mathrm{~s}$ \\
\hline 6 & $4 d^{10} 6 g$ & 43 & $4 d^{9} 6 p 6 f$ & 80 & $4 d^{9} 5 g 8 s$ & 6 & $4 d^{10} 7 p$ & 43 & $4 d^{9} 5 \mathrm{p} 7 \mathrm{~s}$ & 80 & $4 d^{9} 6 \mathrm{p} 7 \mathrm{~d}$ \\
\hline 7 & $4 d^{10} 7 \mathrm{~s}$ & 44 & $4 d^{9} 5 s 6 s$ & 81 & $4 d^{9} 5 \mathrm{~g} 8 \mathrm{~d}$ & 7 & $4 d^{10} 7 f$ & 44 & $4 d^{9} 5 \mathrm{p} 7 \mathrm{~d}$ & 81 & $4 d^{9} 6 \mathrm{p} 7 g$ \\
\hline 8 & $4 d^{10} 7 d$ & 45 & $4 d^{9} 5 \mathrm{~s} 6 \mathrm{~d}$ & 82 & $4 d^{9} 5 g 8 g$ & 8 & $4 d^{10} 8 p$ & 45 & $4 d^{9} 5 \mathrm{p} 7 g$ & 82 & $4 d^{9} 6 d 7 p$ \\
\hline 9 & $4 d^{10} 7 g$ & 46 & $4 d^{9} 5 s 6 g$ & 83 & $4 d^{9} 5 s 9 s$ & 9 & $4 d^{10} 8 f$ & 46 & $4 d^{9} 5 d 7 p$ & 83 & $4 d^{9} 6 d 7 f$ \\
\hline 10 & $4 d^{10} 8 s$ & 47 & $4 d^{9} 5 p 6 p$ & 84 & $4 d^{9} 5 s 9 d$ & 10 & $4 d^{10} 9 p$ & 47 & $4 d^{9} 5 d 7 f$ & 84 & $4 d^{9} 6 f 7 s$ \\
\hline 11 & $4 d^{10} 8 d$ & 48 & $4 d^{9} 5 p 6 f$ & 85 & $4 d^{9} 5 s 9 g$ & 11 & $4 d^{10} 9 f$ & 48 & $4 d^{9} 5 f 7 s$ & 85 & $4 d^{9} 6 f 7 d$ \\
\hline 12 & $4 d^{10} 8 g$ & 49 & $4 d^{9} 5 d 6 s$ & 86 & $4 d^{9} 5 p 9 p$ & 12 & $4 d^{9} 4 f 5 s$ & 49 & $4 d^{9} 5 f 7 d$ & 86 & $4 d^{9} 6 f 7 g$ \\
\hline 13 & $4 d^{10} 9 \mathrm{~s}$ & 50 & $4 d^{9} 5 d 6 d$ & 88 & $4 d^{9} 5 \mathrm{p} 9 \mathrm{f}$ & 13 & $4 d^{9} 4 f 5 d$ & 50 & $4 d^{9} 5 f 7 g$ & 88 & $4 \mathrm{~d}^{10} \varepsilon p$ \\
\hline 14 & $4 d^{10} 9 d$ & 51 & $4 d^{9} 5 d 6 g$ & 89 & $4 d^{9} 5 d 9 s$ & 14 & $4 d^{9} 4 \mathrm{f} 5 g$ & 51 & $4 d^{9} 5 g 7 p$ & 89 & $4 \mathrm{~d}^{10} \varepsilon f$ \\
\hline 15 & $4 d^{10} 9 g$ & 52 & $4 d^{9} 5 f 6 p$ & 90 & $4 d^{9} 5 d_{9} d$ & 15 & $4 d^{9} 4 f 6 s$ & 52 & $4 d^{9} 5 g 7 f$ & 90 & $4 \mathrm{~d}^{10} \varepsilon h$ \\
\hline 16 & $4 d^{9} 4 f^{2}$ & 53 & $4 d^{9} 5 f 6 f$ & 91 & $4 d^{9} 5 d 9 g$ & 16 & $4 d^{9} 4 f 6 d$ & 53 & $4 d^{9} 5 s 8 p$ & 91 & $4 \mathrm{~d}^{10} \varepsilon k$ \\
\hline 17 & $4 d^{9} 5 s^{2}$ & 54 & $4 d^{9} 5 g 6 s$ & 92 & $4 d^{9} 5 f 9 p$ & 17 & $4 d^{9} 4 f 7 s$ & 54 & $4 d^{9} 5 \mathrm{~s} 8 \mathrm{f}$ & 92 & $4 d^{10} \varepsilon m$ \\
\hline 18 & $4 d^{9} 5 p^{2}$ & 55 & $4 d^{9} 5 \mathrm{~g} 6 \mathrm{~d}$ & 93 & $4 d^{9} 5 f 9 f$ & 18 & $4 d^{9} 4 f 7 d$ & 55 & $4 d^{9} 5 p 8 s$ & 93 & $4 \mathrm{~d}^{10} \varepsilon o$ \\
\hline 19 & $4 d^{9} 5 d^{2}$ & 56 & $4 d^{9} 5 \mathrm{~g} 6 \mathrm{~g}$ & 94 & $4 d^{9} 5 g 9 s$ & 19 & $4 d^{9} 4 f 8 s$ & 56 & $4 d^{9} 5 p 8 d$ & & \\
\hline 20 & $4 d^{9} 5 f^{2}$ & 57 & $4 d^{9} 5 \mathrm{~s} 7 \mathrm{~s}$ & 95 & $4 d^{9} 5 \mathrm{~g} 9 \mathrm{~d}$ & 20 & $4 d^{9} 4 f 8 d$ & 57 & $4 d^{9} 5 \mathrm{p} 8 \mathrm{~g}$ & & \\
\hline 21 & $4 d^{9} 5 g^{2}$ & 58 & $4 d^{9} 5 \mathrm{~s} 7 \mathrm{~d}$ & 96 & $4 d^{9} 5 g 9 g$ & 21 & $4 d^{9} 4 f 9 s$ & 58 & $4 d^{9} 5 d 8 p$ & & \\
\hline 22 & $4 d^{9} 6 s^{2}$ & 59 & $4 d^{9} 5 \mathrm{~s} 7 \mathrm{~g}$ & 97 & $4 d^{9} 6 s 7 s$ & 22 & $4 d^{9} 4 f 9 d$ & 59 & $4 d^{9} 5 \mathrm{~d} 8 \mathrm{f}$ & & \\
\hline 23 & $4 d^{9} 6 p^{2}$ & 60 & $4 d^{9} 5 p 7 p$ & 98 & $4 d^{9} 6 s 7 d$ & 23 & $4 d^{9} 5 s 5 p$ & 60 & $4 d^{9} 5 f 8 s$ & & \\
\hline 24 & $4 d^{9} 6 d^{2}$ & 61 & $4 d^{9} 5 \mathrm{p} 7 \mathrm{f}$ & 99 & $4 d^{9} 6 s 7 g$ & 24 & $4 d^{9} 5 \mathrm{~s} 5 \mathrm{f}$ & 61 & $4 d^{9} 5 f 8 d$ & & \\
\hline 25 & $4 d^{9} 6 f^{2}$ & 62 & $4 d^{9} 5 d 7 s$ & 100 & $4 d^{9} 6 p 7 p$ & 25 & $4 d^{9} 5 d 5 p$ & 62 & $4 d^{9} 5 f 8 g$ & & \\
\hline 26 & $4 d^{9} 4 f 5 p$ & 63 & $4 d^{9} 5 d 7 d$ & 101 & $4 d^{9} 6 p 7 f$ & 26 & $4 d^{9} 5 d 5 f$ & 63 & $4 d^{9} 5 g 8 p$ & & \\
\hline 27 & $4 d^{9} 4 f 5 f$ & 64 & $4 d^{9} 5 d 7 g$ & 102 & $4 d^{9} 6 d 7 s$ & 27 & $4 d^{9} 5 p 5 g$ & 64 & $4 d^{9} 5 g 8 f$ & & \\
\hline 28 & $4 d^{9} 4 f 6 p$ & 65 & $4 d^{9} 5 f 7 p$ & 103 & $4 d^{9} 6 d 7 d$ & 28 & $4 d^{9} 5 f 5 g$ & 65 & $4 d^{9} 5 s 9 p$ & & \\
\hline 29 & $4 d^{9} 4 f 6 f$ & 66 & $4 d^{9} 5 f 7 f$ & 104 & $4 d^{9} 6 d 7 g$ & 29 & $4 d^{9} 5 s 6 p$ & 66 & $4 d^{9} 5 s 9 f$ & & \\
\hline 30 & $4 d^{9} 4 f 7 p$ & 67 & $4 d^{9} 5 \mathrm{~g} 7 \mathrm{~s}$ & 105 & $4 d^{9} 6 f 7 p$ & 30 & $4 d^{9} 5 s 6 f$ & 67 & $4 d^{9} 5 p 9 s$ & & \\
\hline 31 & $4 d^{9} 4 f 7 f$ & 68 & $4 d^{9} 5 \mathrm{~g} 7 \mathrm{~d}$ & 106 & $4 d^{9} 6 f 7 f$ & 31 & $4 d^{9} 5 p 6 s$ & 68 & $4 d^{9} 5 p 9 d$ & & \\
\hline 32 & $4 d^{9} 4 f 8 p$ & 69 & $4 d^{9} 5 \mathrm{~g} 7 \mathrm{~g}$ & 107 & $4 d^{10} \varepsilon s$ & 32 & $4 d^{9} 5 \mathrm{p} 6 \mathrm{~d}$ & 69 & $4 d^{9} 5 \mathrm{p} 9 \mathrm{~g}$ & & \\
\hline 33 & $4 d^{9} 4 f 8 f$ & 70 & $4 d^{9} 5 s 8 s$ & 108 & $4 \mathrm{~d}^{10} \varepsilon d$ & 33 & $4 d^{9} 5 \mathrm{p} 6 g$ & 70 & $4 d^{9} 5 d 9 p$ & & \\
\hline 34 & $4 d^{9} 4 f 8 p$ & 71 & $4 d^{9} 5 s 8 d$ & 109 & $4 d^{10} \varepsilon \mathrm{g}$ & 34 & $4 d^{9} 5 \mathrm{~d} 6 \mathrm{p}$ & 71 & $4 d^{9} 5 d 9 f$ & & \\
\hline 35 & $4 d^{9} 4 f 8 f$ & 72 & $4 d^{9} 5 \mathrm{~s} 8 \mathrm{~g}$ & 110 & $4 \mathrm{~d}^{10} \varepsilon i$ & 35 & $4 d^{9} 5 \mathrm{~d} 6 \mathrm{f}$ & 72 & $4 d^{9} 5 f 9 s$ & & \\
\hline 36 & $4 d^{9} 5 \mathrm{~s} 5 \mathrm{~d}$ & 73 & $4 d^{9} 5 p 8 p$ & 111 & $4 d^{10} \varepsilon l$ & 36 & $4 d^{9} 5 f 6 s$ & 73 & $4 d^{9} 5 f 9 d$ & & \\
\hline 37 & $4 d^{9} 5 \mathrm{~s} 5 \mathrm{~g}$ & 74 & $4 d^{9} 5 p 8 f$ & 112 & $4 d^{10} \varepsilon n$ & 37 & $4 d^{9} 5 f 6 d$ & 74 & $4 d^{9} 5 f 9 g$ & & \\
\hline
\end{tabular}

University of Texas at Austin by Sugar et al [33]. Three strong doublets of the Ag I isoelectronic sequence were identified: $4 \mathrm{~d}^{10} 4 \mathrm{f}-4 \mathrm{~d}^{9} 4 \mathrm{f}\left({ }^{1} \mathrm{P}\right) 4 \mathrm{f}{ }^{2} \mathrm{D},{ }^{2} \mathrm{~F},{ }^{2} \mathrm{G}$. Interpolated and extrapolated wavelengths in this sequence were derived from plots of the difference between observed and calculated values for the transition energies [33]. Churilov and Joshi [34] classified two $\mathrm{Xe}^{7+}$ resonance lines $\left(4 \mathrm{~d}^{10} 5 \mathrm{~s}-4 \mathrm{~d}^{9} 5 \mathrm{~s} 4 \mathrm{f}\right)$.

In this paper, we evaluate the total DR rate coefficient of Pd-like tungsten $\left(\mathrm{W}^{28+}\right)$ since this is one of the dominant charge states in the present-day tokamaks, as mentioned above. We also evaluate the dielectronic recombination rate coefficients for the first excited odd- and even-parity states in Ag-like tungsten $\left(\mathrm{W}^{27+}\right)$. The present level-bylevel calculations include the DR contributions of all of the levels (over 17000) in the Ag-like inner-shell excited configurations $[\mathrm{Kr}] 4 \mathrm{~d}^{9} 4 \mathrm{f} n l(n=4-9),[\mathrm{Kr}] 4 \mathrm{~d}^{9} 5 l^{\prime} n l,(n=$ 5-9) and $[\mathrm{Kr}] 4 \mathrm{~d}^{9} 6 l^{\prime} n l(n=6-7)$. Energy levels, radiative transition probabilities and autoionization rates for those states are calculated using the HULLAC code, the COWAN code and the RMBPT code. These three codes allow us to check the accuracy of our calculations and to achieve confidence that our predictions are reliable. This paper continues our efforts on the calculation of the DR rate coefficients that were previously determined for C I [36], C II [37, 38], C III [39], O IV [40], O V [41], Ne VII [42], Ne VI [43], Mg-like Fe [44], Mg-like Zn, Kr, and Mo [45], Ag-like Xe [46], Na-like tungsten [14] and Mglike tungsten [15]. In the following, we omit the core $[\mathrm{Kr}]=$ $1 s^{2} 2 s^{2} 2 p^{6} 3 s^{2} 3 p^{6} 3 d^{10} 4 s^{2} 4 p^{6}$ from the configuration notation.

\section{Energy levels, transition probabilities and autoionization rates}

Detailed calculations of DR parameters should include the determination of such characteristics as energies, radiative transition probabilities and autoionization rates for atomic states in the recombined ion. The atomic structure of Aglike tungsten was represented by 112 even-parity and 93 oddparity configurations $4 \mathrm{~d}^{10} n l$ with $n=4-9,4 \mathrm{~d}^{9} 4 \mathrm{f} n l$ with $n=$ 4-9, $4 \mathrm{~d}^{9} 5 l^{\prime} n l^{\prime \prime}$ with $n=5-9$ and $4 \mathrm{~d}^{9} 6 l^{\prime} n l^{\prime \prime}$ with $n=6-7$ (see table 1), so that the total number of energy levels is 8620 and 7930 for even and odd parities, respectively. 
Table 2. Energies $\left(E\right.$ in $10^{3} \mathrm{~cm}^{-1}$ ) calculated to the first, second, third and all orders of RMBPT for the $4 \mathrm{~d}^{10} n l^{2} L_{j}$ states of Ag-like W. Comparison of RMBPT, COWAN and HULLAC results.

\begin{tabular}{|c|c|c|c|c|c|c|}
\hline Level & $E^{\text {(COWAN) }}$ & $E^{(\text {HULLAC })}$ & $E^{(\mathrm{DF})}$ & $E^{(\mathrm{DF}+2)}$ & $E^{(\mathrm{DF}+2+3)}$ & $E^{(\mathrm{DF}+\mathrm{SD})}$ \\
\hline${ }^{2} \mathrm{~F}_{5 / 2}$ & 0.000 & 0.000 & 0.000 & 0.000 & 0.000 & 0.000 \\
\hline${ }^{2} \mathrm{~F}_{7 / 2}$ & 30.349 & 28.568 & 30.338 & 29.666 & 29.552 & 29.555 \\
\hline $5 s^{2} S_{1 / 2}$ & 1120.817 & 1116.214 & 1110.458 & 1123.119 & 1122.921 & 1122.912 \\
\hline $5 \mathrm{p}^{2} \mathrm{P}_{1 / 2}$ & 1474.075 & 1471.214 & 1463.973 & 1476.099 & 1475.952 & 1475.928 \\
\hline $5 \mathrm{p}^{2} \mathrm{P}_{3 / 2}$ & 1699.138 & 1693.351 & 1688.633 & 1700.510 & 1700.094 & 1700.068 \\
\hline $5 d^{2} D_{3 / 2}$ & 2267.708 & 2261.046 & 2258.621 & 2276.390 & 2248.483 & \\
\hline $5 d^{2} D_{5 / 2}$ & 2312.498 & 2305.891 & 2304.559 & 2322.042 & 2314.743 & \\
\hline $5 f^{2} F_{5 / 2}$ & 2983.307 & 2973.445 & 2973.554 & 2979.276 & 2977.032 & \\
\hline $5 f{ }^{2} F_{7 / 2}$ & 2994.422 & 2983.528 & 2984.288 & 2990.111 & 2987.210 & \\
\hline $6 s{ }^{2} S_{1 / 2}$ & 3387.836 & 3378.885 & 3373.064 & 3391.977 & 3389.232 & \\
\hline $6 \mathrm{p}^{2} \mathrm{P}_{1 / 2}$ & 3575.025 & 3559.938 & 3552.703 & 3581.045 & 3575.828 & \\
\hline $6 \mathrm{p}^{2} \mathrm{P}_{3 / 2}$ & 3686.519 & 3669.224 & 3663.367 & 3690.479 & 3666.069 & \\
\hline $6 d^{2} D_{3 / 2}$ & 3979.768 & 3962. & 3956.975 & 3987.900 & 3977.340 & \\
\hline $6 \mathrm{~d}^{2} \mathrm{D}_{5 / 2}$ & 4004.424 & 55 & 3980.467 & 4014.900 & 3999.254 & \\
\hline $7 \mathrm{~s}^{2} \mathrm{~S}_{1 / 2}$ & 4568.310 & 4 & 4544.174 & 4573.655 & 456 & \\
\hline $6 \mathrm{~g}^{2} \mathrm{G}_{7 / 2}$ & 4618.305 & 4597.012 & 4592.695 & 4621.978 & 4616.123 & \\
\hline $6 \mathrm{~g}^{2} \mathrm{G}_{9 / 2}$ & 4619.900 & 4598.040 & 4593.885 & 4623.263 & 4617.326 & \\
\hline $7 \mathrm{p}^{2} \mathrm{P}_{1 / 2}$ & 4670.953 & 4659.018 & 4647.983 & 4678.162 & 4672.763 & \\
\hline $7 \mathrm{p}^{2} \mathrm{P}_{3 / 2}$ & 4735.382 & 4721.059 & 4711.247 & 4741.837 & 4735.921 & \\
\hline $7 \mathrm{~d}^{2} \mathrm{D}_{3 / 2}$ & 4903.066 & 4893.714 & 4882.853 & 4910.078 & 4902.607 & \\
\hline $7 d^{2} D_{5 / 2}$ & 4916.581 & 4906.989 & 4896.615 & 4926.576 & 4919.576 & \\
\hline $7 f^{2} F_{5 / 2}$ & 5119.880 & 5107.947 & 5095.784 & 5121.462 & 5063.196 & \\
\hline $7 f^{2} F_{7 / 2}$ & 5123.904 & 5110.978 & 5099.295 & 5127.418 & 5114.266 & \\
\hline $7 \mathrm{~g}^{2} \mathrm{G}_{7 / 2}$ & 5281.651 & 5268.842 & 5255.163 & 5288.799 & 5278.965 & \\
\hline $7 \mathrm{~g}^{2} \mathrm{G}_{9 / 2}$ & 5282.743 & 5269.545 & 5256.048 & 5290.004 & 5280.384 & \\
\hline Ionization potential & & & 7075.535 & 7111.369 & 7105.273 & 7105.516 \\
\hline
\end{tabular}

With almost 10000 levels per parity, the total number of radiative transitions is in the tens of millions. To reduce the computational load, we neglected transitions with small probabilities, $A_{r}<10^{5} \mathrm{~s}^{-1}$ (the strongest transitions, as will be shown below, have $A_{r}$ on the order of $10^{8} \mathrm{~s}^{-1}$ ). Even with this restriction, the resulting list of radiative transitions within the Ag-like ion includes about $2 \times 10^{6}$ transitions.

Our large-scale calculations of atomic properties are based on the previously mentioned three atomic computer codes: the code developed by Cowan, which uses a quasi-relativistic Hartree-Fock method with superposition of configurations (in the following we will refer to it as the 'COWAN' code) [47], the multi-configuration relativistic HULLAC code [48] and the RMBPT code. The RMBPT computations were carried out with two methods: (i) the relativistic second- and thirdorder, and all-order RMBPT [49, 50] for singly excited states, and (ii) the relativistic second order for doubly excited states $[51,52]$.

We use the version of the Cowan code available in [53]. That version allows us to take into account a large number of configurations (up to 100 odd- and 100 even-parity configurations). The scaling of electrostatic integrals in the Cowan code allows one to correct for the correlation effects and to obtain good agreement with experimental energies. We employed a single scaling factor $(0.85)$ for all electrostatic integrals.

In the HULLAC code, the intermediate-coupling level energies are calculated using the relativistic version of the parametric potential method, including configuration mixing. The autoionization rate coefficients are calculated in the distorted-wave approximation, implementing the highly efficient factorization-interpolation method.

The RMBPT code, including the Breit interaction, is used to evaluate energies and transition rates for multipole transitions. This method is based on the RMPBT, agrees with multi-configuration Dirac-Fock (MCDF) calculations in the lowest order, includes all second-order correlation corrections and includes corrections from negative energy states. Firstorder perturbation theory is used to obtain intermediatecoupling coefficients, and second-order RMBPT is used to determine the matrix elements. The RMBPT code is an $a b$ initio code without the scaling of electrostatic integrals as used in the Cowan code and without any parametric potentials as employed in the HULLAC code.

The results of energy calculations for the singly excited $4 \mathrm{~d}^{10} \mathrm{nl}$ states of $\mathrm{W}^{27+}$ are summarized in table 2, which presents the lowest-order Dirac-Fock (DF) energies $E^{\mathrm{DF}}$, the second-order $\left(E^{\mathrm{DF}+2}\right)$ and third-order Coulomb correlation energies $\left(E^{\mathrm{DF}+2+3}\right)$, the all-order 'SD' energies $E^{\mathrm{DF}+\mathrm{SD}}$, as well as the COWAN and HULLAC code results, $E^{\text {(COWAN) }}$ and $E^{\text {(HULLAC) }}$, respectively. The last line of table 2 presents the RMBPT value for the ionization potential of $\mathrm{W}^{27+}$. The differences between all results are about $0.1-1 \%$, except for the $4 \mathrm{~d}^{10} 4 \mathrm{f}{ }^{2} \mathrm{~F}$ splitting. One can see from table 2 that the RMBPT values of the $4 \mathrm{~d}^{10} 4 \mathrm{f}^{2} \mathrm{~F}$ splitting agree with each other except the $E^{(\mathrm{DF})}$ value; however, the $E^{(\mathrm{DF})}$ value agrees with $E^{\text {(COWAN) }}$ and with the recommended NIST data [24]. Only a few values of the all-order SD energies $E^{(\mathrm{DF}+\mathrm{SD})}$ are given in table 2, because of a convergence problem. This convergence 
Table 3. Wavelengths $\lambda$ (in $\AA$ ), and weighted transition rates $\left(g A_{r}\right)$ for the $4 \mathrm{~d}^{10} n l_{1}-4 \mathrm{~d}^{10} n l_{2}$ transitions of $\mathrm{Ag}$-like tungsten. Comparison of results calculated by the RMBPT, Cowan and HULLAC codes.

\begin{tabular}{|c|c|c|c|c|c|c|c|c|}
\hline \multirow[b]{2}{*}{$n l, L S J$} & \multirow[b]{2}{*}{$n l, L S J$} & \multicolumn{3}{|c|}{ Wavelengths in $\lambda(\AA)$} & \multicolumn{4}{|c|}{ Weighted transition rates $g A_{r}$ in $\mathrm{s}^{-1}$} \\
\hline & & $\overline{\mathrm{RMBPT}}$ & COWAN & HULLAC & $\overline{\mathrm{RMBPT}}$ & $\mathrm{DF}$ & COWAN & $\overline{\text { HULLAC }}$ \\
\hline $4 \mathrm{f}^{2} \mathrm{~F}_{5 / 2}$ & $7 \mathrm{~g}^{2} \mathrm{G}_{7 / 2}$ & 18.93 & 18.93 & 18.98 & $1.66[13]$ & $1.64[13]$ & $1.80[13]$ & $2.00[13]$ \\
\hline $4 \mathrm{f}^{2} \mathrm{~F}_{7 / 2}$ & $7 \mathrm{~g}^{2} \mathrm{G}_{9 / 2}$ & 19.03 & 19.04 & 19.08 & $2.15[13]$ & $2.10[13]$ & $2.15[13]$ & $2.56[13]$ \\
\hline $4 \mathrm{f}^{2} \mathrm{~F}_{5 / 2}$ & $6 \mathrm{~g}^{2} \mathrm{G}_{7 / 2}$ & 21.66 & 21.65 & 21.75 & $2.42[13]$ & $2.28[13]$ & $2.98[13]$ & $2.49[13]$ \\
\hline $4 \mathrm{f}^{2} \mathrm{~F}_{7 / 2}$ & $6 g^{2} G_{9 / 2}$ & 21.80 & 21.79 & 21.88 & $3.18[13]$ & $2.92[13]$ & $3.85[13]$ & $3.19[13]$ \\
\hline $4 \mathrm{f}^{2} \mathrm{~F}_{7 / 2}$ & $6 \mathrm{~d}^{2} \mathrm{D}_{5 / 2}$ & 25.13 & 25.16 & 25.27 & $1.52[11]$ & $7.37[11]$ & $3.74[11]$ & $9.02[11]$ \\
\hline $4 \mathrm{f}^{2} \mathrm{~F}_{7 / 2}$ & $5 \mathrm{~g}^{2} \mathrm{G}_{9 / 2}$ & 28.55 & 28.52 & 28.74 & $5.22[13]$ & $5.18[13]$ & $6.06[13]$ & $5.27[13]$ \\
\hline $4 \mathrm{f}^{2} \mathrm{~F}_{7 / 2}$ & $5 \mathrm{~g}^{2} \mathrm{G}_{7 / 2}$ & 28.57 & 28.55 & 28.75 & $1.70[12]$ & $1.48[12]$ & $1.77[12]$ & $1.50[12]$ \\
\hline $5 \mathrm{p}^{2} \mathrm{P}_{1 / 2}$ & $7 \mathrm{~s}^{2} \mathrm{~S}_{1 / 2}$ & 32.34 & 32.32 & 32.44 & $2.49[11]$ & $3.15[11]$ & $3.32[11]$ & $3.96[11]$ \\
\hline $5 \mathrm{p}^{2} \mathrm{P}_{3 / 2}$ & $7 s^{2} S_{1 / 2}$ & 34.87 & 34.85 & 34.96 & $6.54[11]$ & $7.56[11]$ & $5.10[11]$ & $9.00[11]$ \\
\hline $5 f^{2} \mathrm{~F}_{5 / 2}$ & $7 \mathrm{~g}^{2} \mathrm{G}_{7 / 2}$ & 43.30 & 43.51 & 43.56 & $4.76[12]$ & $3.97[12]$ & $4.36[12]$ & $5.04[12]$ \\
\hline $5 \mathrm{f}^{2} \mathrm{~F}_{7 / 2}$ & $7 \mathrm{~g}^{2} \mathrm{G}_{9 / 2}$ & 43.48 & 43.70 & 43.74 & $6.34[12]$ & $5.18[12]$ & $5.77[12]$ & $6.54[12]$ \\
\hline $5 f^{2} \mathrm{~F}_{7 / 2}$ & $7 \mathrm{~g}^{2} \mathrm{G}_{7 / 2}$ & 43.50 & 43.72 & 43.76 & $1.82[11]$ & $1.48[11]$ & $1.63[11]$ & $1.87[11]$ \\
\hline $4 \mathrm{f}^{2} \mathrm{~F}_{5 / 2}$ & $5 \mathrm{~d}^{2} \mathrm{D}_{3 / 2}$ & 44.04 & 44.10 & 44.23 & $1.67[11]$ & $1.33[12]$ & $2.62[11]$ & $1.42[12]$ \\
\hline $5 \mathrm{p}^{2} \mathrm{P}_{3 / 2}$ & $6 s^{2} S_{1 / 2}$ & 59.31 & 59.22 & 59.33 & $1.56[12]$ & $1.47[12]$ & $1.69[12]$ & $1.51[12]$ \\
\hline $5 \mathrm{f}^{2} \mathrm{~F}_{5 / 2}$ & $6 \mathrm{~g}^{2} \mathrm{G}_{7 / 2}$ & 60.88 & 61.16 & 61.59 & $3.84[12]$ & $3.86[12]$ & $2.78[12]$ & $4.12[12]$ \\
\hline $5 f^{2} \mathrm{~F}_{7 / 2}$ & $6 g^{2} \mathrm{G}_{9 / 2}$ & 61.23 & 61.52 & 61.94 & $5.00[12]$ & $5.08[12]$ & $4.87[12]$ & $5.43[12]$ \\
\hline $5 \mathrm{f}^{2} \mathrm{~F}_{7 / 2}$ & $6 \mathrm{~g}^{2} \mathrm{G}_{7 / 2}$ & 61.28 & 61.58 & 61.98 & $1.43[11]$ & $1.46[11]$ & $1.23[11]$ & $1.56[11]$ \\
\hline $5 f{ }^{2} F_{5 / 2}$ & $6 \mathrm{~d}^{2} \mathrm{D}_{5 / 2}$ & 96.56 & 97.93 & 98.81 & $4.91[10]$ & $4.43[10]$ & $5.39[10]$ & $4.70[10]$ \\
\hline $5 \mathrm{f}^{2} \mathrm{~F}_{5 / 2}$ & $6 d^{2} \mathrm{D}_{3 / 2}$ & 99.14 & 100.36 & 101.10 & $7.27[11]$ & $6.39[11]$ & $7.08[11]$ & $6.77[11]$ \\
\hline $5 f{ }^{2} F_{5 / 2}$ & $5 \mathrm{~d}^{2} \mathrm{D}_{3 / 2}$ & 142.28 & 139.74 & 140.37 & $8.93[11]$ & $1.37[12]$ & $1.07[12]$ & $1.35[12]$ \\
\hline $5 p^{2} P_{3 / 2}$ & $5 d^{2} D_{5 / 2}$ & 162.37 & 163.04 & 163.25 & $6.05[11]$ & $8.28[11]$ & $6.13[11]$ & $8.14[11]$ \\
\hline $5 \mathrm{p}^{2} \mathrm{P}_{3 / 2}$ & $5 s^{2} S_{1 / 2}$ & 173.23 & 172.91 & 173.27 & $2.48[11]$ & $3.37[11]$ & $2.52[11]$ & $3.34[11]$ \\
\hline $5 f^{2} \mathrm{~F}_{5 / 2}$ & $5 \mathrm{~g}^{2} \mathrm{G}_{7 / 2}$ & 179.79 & 181.87 & 187.43 & $5.86[11]$ & $7.57[11]$ & $7.24[11]$ & $7.35[11]$ \\
\hline $5 \mathrm{f}^{2} \mathrm{~F}_{7 / 2}$ & $5 \mathrm{~g}^{2} \mathrm{G}_{7 / 2}$ & 183.36 & 185.62 & 191.04 & $1.94[10]$ & $2.63[10]$ & $2.47[10]$ & $2.56[10]$ \\
\hline $5 \mathrm{p}^{2} \mathrm{P}_{1 / 2}$ & $5 \mathrm{~s}^{2} \mathrm{~S}_{1 / 2}$ & 283.18 & 283.08 & 281.69 & $2.79[10]$ & $3.78[10]$ & $2.91[10]$ & $3.82[10]$ \\
\hline
\end{tabular}

problem of the all-order iterative scheme for the $n \mathrm{~d}$ states was discussed in [54].

In table 3 , we present wavelengths $\lambda$ (in $\AA$ ), and weighted radiative transition rates $g A_{r}$ for the $4 \mathrm{~d}^{10} n_{1} l_{1}-4 \mathrm{~d}^{10} n_{2} l_{2}$ transitions of Ag-like tungsten. Results for wavelength and transition rates are obtained from the three codes, RMBPT (columns 3, 6 and 7), COWAN (columns 4 and 8) and HULLAC (columns 5 and 9). The electric-dipole-reduced matrix elements used to evaluate line strengths, oscillator strengths and radiative transition rates in $\mathrm{W}^{27+}$ (column 6 of table 3 ) are calculated using the form-independent third-order perturbation theory developed by Savukov and Johnson [55]. The good precision of this method was demonstrated for alkalimetal atoms [55] and later for Ag-like ions [50]. Numerical results for the oscillator strengths in Ag-like ions with $Z=47-$ 60 were presented for the $5 \mathrm{~s}_{1 / 2}-5 \mathrm{p}_{j}, 5 \mathrm{p}_{j}-5 \mathrm{~d}_{j^{\prime}}, 4 \mathrm{f}_{j}-5 \mathrm{~d}_{j^{\prime}}$, and $4 \mathrm{f}_{j}-5 g_{j^{\prime}}$ transitions in [50]. It was emphasized in [50] that the second- and third-order line strength results obtained by that method were gauge independent while the first-order results were gauge dependent. In column 7 of table 3, we present RMBPT results in the lowest-order (DF) approximation. It should be noted that these lowest-order RMBPT values agree with the results evaluated in the MCDF approach.

In our study, we have evaluated the complete set of $n f_{j}-n^{\prime} d_{j^{\prime}}, n f_{j}-n^{\prime} g_{j^{\prime}}$ transitions with $n=4-7, n^{\prime}=5-7$ and $n p_{j}-n^{\prime} s_{j^{\prime}}, n p_{j}-n^{\prime} d_{j^{\prime}}$ transitions with $n=5-7, n^{\prime}=5-7$. We compare wavelengths and transition rates obtained by RMBPT, COWAN and HULLAC codes. A few transitions, listed in table 3, illustrate our work. The difference in the wavelengths is about $0.1-0.5 \%$, while the smallest difference in transition rates is about $10-30 \%$. There are transitions with a difference in $g A_{r}$ as large as a factor of 2-3 (see, for example, the $g A_{r}$ values given in table 3 for the $4 \mathrm{f}^{2} \mathrm{~F}_{7 / 2}-6 \mathrm{~d}{ }^{2} \mathrm{D}_{5 / 2}$ transition). Such a difference is due to very large contributions of correlation effects in the dipole matrix elements. Those large contributions of relativistic effects for transitions involving $4 \mathrm{f}_{j}$ and $5 \mathrm{f}_{j}$ states were discussed in $[25,50]$.

Relativistic many-body calculations of excitation energies and transition rates from core-excited states in silver-like ions were presented in [28]. The detailed discussion of the various contributions to the dipole matrix elements and energy levels for core-excited states was also included for silver-like tungsten. Additionally, calculations of energies and radiative transition rates for Ag-like $\mathrm{W}$ were performed with the COWAN and HULLAC codes to compare with results from the RMBPT code [28]. Because those results already exist, we do not present below any tabulated values for wavelengths and radiative transition rates involving core-excited states. Instead, we present below only results for autoionizing rates involving core-excited states, which were not considered in [28].

Energies and weighted autoionizing rates $\left(g A_{a}\right)$ for the core-excited $4 \mathrm{~d}^{9} 5 \mathrm{f}^{2}$ states calculated using RMBPT, COWAN and HULLAC codes are given in table 4. We use $j j$ designations to label levels in the first column of table 4, i.e. $5 \mathrm{f}_{j} 5 \mathrm{f}_{j^{\prime}}\left(J_{12}\right) 4 \mathrm{~d}_{j^{\prime \prime}}(J)$, where $J_{12}$ is the angular momentum combining two $5 \mathrm{f}_{j}$ one-electron states and $J$ is the angular momentum combining two $5 \mathrm{f}_{j}$ one-electron states and one hole $4 \mathrm{~d}$ state (for details, see [28]). There are 107 states with different $j, j^{\prime}, j^{\prime \prime}, J_{12}$ and $J$. We evaluated energies and 
Table 4. Energies ( $E$ in $\left.10^{3} \mathrm{~cm}^{-1}\right)$ and weighted autoionizing rates $\left(g A_{a}\right.$ in s $\left.{ }^{-1}\right)$ for the core-excited $4 \mathrm{~d}^{9} 5 \mathrm{f}^{2}$ levels calculated using the RMBPT, COWAN and HULLAC codes.

\begin{tabular}{|c|c|c|c|c|c|c|c|}
\hline Level & $E^{(\mathrm{RMBPT})}$ & $E^{\text {(COWAN) }}$ & $E^{(\text {HULLAC })}$ & $g A_{a}^{(\mathrm{DF})}$ & $g A_{a}^{(\mathrm{MP})}$ & $g A_{a}^{\text {(COWAN) }}$ & $g A_{a}^{(\text {HULLAC })}$ \\
\hline $5 f_{5 / 2} 5 f_{7 / 2}(3) 4 d_{5 / 2}(1 / 2)$ & 7665.8 & 7697.4 & 7706.3 & $2.77[11]$ & $2.28[11]$ & $1.58[11]$ & $6.48[10]$ \\
\hline $5 f_{7 / 2} 5 f_{7 / 2}(4) 4 d_{5 / 2}(13 / 2)$ & 7666.8 & 7700.3 & 7706.0 & $3.53[13]$ & $2.69[13]$ & $4.00[13]$ & $4.69[12]$ \\
\hline $5 f_{5 / 2} 5 f_{7 / 2}(5) 4 d_{5 / 2}(11 / 2)$ & 7667.2 & 7699.8 & 7707.2 & $3.77[11]$ & $3.78[11]$ & $1.75[12]$ & $4.00[11]$ \\
\hline $5 f_{5 / 2} 5 f_{7 / 2}(3) 4 d_{5 / 2}(3 / 2)$ & 7669.2 & 7700.3 & 7709.9 & $2.24[13]$ & $1.59[13]$ & $2.30[13]$ & $7.40[12]$ \\
\hline $5 f_{7 / 2} 5 f_{7 / 2}(4) 4 d_{5 / 2}(11 / 2)$ & 7671.2 & 7703.7 & 7711.6 & $4.49[11]$ & $3.12[11]$ & $2.33[12]$ & $5.23[11]$ \\
\hline $5 f_{7 / 2} 5 f_{7 / 2}(4) 4 d_{5 / 2}(5 / 2)$ & 767 & 77 & 77 & 3] & 3] & [13] & $4[12]$ \\
\hline $5 f_{5 / 2} 5 f_{7 / 2}(2) 4 d_{5 / 2}(3 / 2)$ & 7684.3 & 7712.7 & 7725.5 & $2.38[13]$ & $1.71[13]$ & $3.93[13]$ & $1.18[13]$ \\
\hline $5 f_{5 / 2} 5 f_{7 / 2}(6) 4 d_{5 / 2}(11 / 2)$ & 7690.1 & 7723.7 & 7736.6 & $2.17[13]$ & $1.65[13]$ & $2.56[13]$ & $3.21[12]$ \\
\hline $5 f_{5 / 2} 5 f_{5 / 2}(0) 4 d_{5 / 2}(5 / 2)$ & 7695.2 & 7724.2 & 7738.7 & $5.09[12]$ & $3.86[12]$ & $4.20[12]$ & $6.27[11]$ \\
\hline $5 f_{5 / 2} 5 f_{7 / 2}(6) 4 d_{5 / 2}(13 / 2)$ & 7696.8 & 7730.0 & 7744.1 & $7.60[12]$ & $6.36[12]$ & $3.72[13]$ & $3.90[12]$ \\
\hline $5 \mathrm{f}_{5 / 2} 5 \mathrm{f}_{7 / 2}(1) 4 \mathrm{~d}_{5 / 2}(5 / 2)$ & 7700.9 & 7729.3 & 7743.5 & $2.07[13]$ & $1.56[13]$ & $3.78[13]$ & $6.15[12]$ \\
\hline $5 f_{7 / 2} 5 f_{7 / 2}(2) 4 d_{5 / 2}(1 / 2)$ & 7705.4 & 7735.8 & 7749.4 & $5.22[12]$ & $4.30[12]$ & $2.04[12]$ & $7.10[11]$ \\
\hline $5 \mathrm{f}_{7 / 2} 5 \mathrm{f}_{7 / 2}(0) 4 \mathrm{~d}_{5 / 2}(5 / 2)$ & 7741.2 & 7766.6 & 7787.7 & $2.46[13]$ & $2.00[13]$ & $2.25[13]$ & $6.47[12]$ \\
\hline $5 f_{5 / 2} 5 f_{7 / 2}(3) 4 d_{3 / 2}(3 / 2)$ & 7788.4 & 7818.5 & 7825.6 & $9.65[12]$ & $7.23[12]$ & $1.03[13]$ & $1.61[12]$ \\
\hline $5 f_{5 / 2} 5 f_{5 / 2}(2) 4 d_{3 / 2}(3 / 2)$ & 7794.4 & 7824.5 & 7833.5 & $1.67[13]$ & $1.24[13]$ & $1.96[13]$ & $6.08[12]$ \\
\hline $5 f_{5 / 2} 5 f_{7 / 2}(3) 4 d_{3 / 2}(5 / 2)$ & 7796.7 & 7828.5 & 7835.6 & $7.83[12]$ & $5.50[12]$ & $3.65[13]$ & $6.10[12]$ \\
\hline $5 f_{7 / 2} 5 f_{7 / 2}(4) 4 d_{3 / 2}(5 / 2)$ & 7804.0 & 7834.3 & 7841.8 & $3.19[13]$ & $2.44[13]$ & $1.17[13]$ & $4.46[12]$ \\
\hline $5 f_{7 / 2} 5 f_{7 / 2}(4) 4 d_{3 / 2}(9 / 2)$ & 7809.5 & 7842.2 & 7850.7 & $3.59[12]$ & $4.37[11]$ & $3.42[12]$ & $6.53[11]$ \\
\hline $5 f_{5 / 2} 5 f_{7 / 2}(2) 4 d_{3 / 2}(3 / 2)$ & 7809.6 & 7838.9 & 7850.2 & $1.19[13]$ & $8.86[12]$ & $1.72[13]$ & $7.24[12]$ \\
\hline $5 f_{5 / 2} 5 f_{7 / 2}(2) 4 d_{3 / 2}(5 / 2)$ & 7811.2 & 7840.4 & 7851.6 & $1.60[13]$ & $1.16[13]$ & $2.55[13]$ & $5.59[12]$ \\
\hline $5 f_{7 / 2} 5 f_{7 / 2}(2) 4 d_{3 / 2}(3 / 2)$ & 7816.3 & 7845.4 & 7856.5 & $3.04[12]$ & $2.43[12]$ & $9.57[11]$ & $8.96[11]$ \\
\hline $5 f_{5 / 2} 5 f_{7 / 2}(6) 4 d_{3 / 2}(11 / 2)$ & 7823.5 & 7856.4 & 7868.9 & $2.37[13]$ & $1.77[13]$ & $1.94[13]$ & $2.16[12]$ \\
\hline $5 \mathrm{f}_{5 / 2} 5 \mathrm{f}_{7 / 2}(1) 4 \mathrm{~d}_{3 / 2}(3 / 2)$ & 7824.2 & 7852.7 & 7866.4 & $2.14[12]$ & $1.75[12]$ & $2.49[12]$ & $7.55[11]$ \\
\hline $5 f_{7 / 2} 5 f_{7 / 2}(2) 4 d_{3 / 2}(1 / 2)$ & 7837.6 & 7866.6 & 7879.8 & $3.63[12]$ & $3.03[12]$ & $1.50[12]$ & $4.90[11]$ \\
\hline $5 f_{7 / 2} 5 f_{7 / 2}(0) 4 d_{3 / 2}(3 / 2)$ & 7874.2 & 7898.5 & 7919.9 & $1.66[13]$ & $1.36[13]$ & $9.80[12]$ & $4.71[12]$ \\
\hline
\end{tabular}

weighted autoionizing rates for all 107 states. However, only selected results of our calculations are listed to illustrate our work. One can see that the energies of the $5 \mathrm{f}_{j} 5 \mathrm{f}_{j^{\prime}}\left(J_{12}\right) 4 \mathrm{~d}_{j^{\prime \prime}}(J)$ states are larger than the energy of the $4 \mathrm{~d}^{10}$ threshold given in the last line of table 2. This makes those states autoionizing states. Autoionization rates are calculated using both DiracFock and model-potential wavefunctions (columns $g A_{a}^{(\mathrm{DF})}$ and $g A_{a}^{(\mathrm{MP})}$ in table 4). A detailed comparison of the 'DF' and 'MP' approaches was given by Safronova et al in [56]. There is a substantial difference (from $30-50 \%$ up to a factor of 2-10) among the results given in the last four columns of table 4.

The disagreement among the results calculated by the COWAN and HULLAC codes for $g A_{a}$ for $\mathrm{W}^{63+}$ was discussed by Safronova et al in [14]. In the case of the COWAN code, the $g A_{a}$ values were calculated with an input of only one energy value for the free electron wavefunctions. In [14] the dependence of the $g A_{a}$ on the input energies (200 and 700 Ry) was checked. The difference was about a factor of 2-5 for most cases. The $g A_{a}$ values obtained by the HULLAC code were found to lie between the values obtained by the COWAN code for values of $200 \mathrm{Ry}$ and $700 \mathrm{Ry}$ for the free electron energy [14]. When calculating the $g A_{a}^{(\mathrm{DF})}$ and $g A_{a}^{(\mathrm{MP})}$ values, we obtain the energy value for the free electron from the energy of the one-electron Dirac-Fock and the model-potential wavefunctions. For example, for the $5 \mathrm{f}_{j_{1}} 5 \mathrm{f}_{j_{2}} \rightarrow 4 \mathrm{~d}_{j_{3}} \varepsilon_{j_{4}}$ decay, the energy $E\left(\varepsilon_{j_{4}}\right)$ is equal to $\left[E\left(5 \mathrm{f}_{j_{1}}\right)+E\left(5 \mathrm{f}_{j_{2}}\right)-E\left(4 \mathrm{~d}_{j_{3}}\right)\right]$. Using Dirac-Fock energies, we find that the range of $E\left(\varepsilon_{j_{4}}\right)$ is $1068000-913000 \mathrm{~cm}^{-1}$ in the $\mathrm{W}^{27+}$ ion. Using modelpotential energies, we find this range of energies to be 366000 $218000 \mathrm{~cm}^{-1}$. These energies could be used to evaluate the Dirac-Fock functions and the model-potential functions for the free electron and subsequently the $g A_{a}^{(\mathrm{DF})}$ and $g A_{a}^{(\mathrm{MP})}$ values. A large difference in the free-electron energy leads to a large difference in the results given in columns $g A_{a}^{(\mathrm{DF})}$ and $g A_{a}^{(\mathrm{MP})}$ of table 4.

The energies of the $4 d^{9} 5 f^{2}$ levels given in table 4 are in the range of $7874000-7655000 \mathrm{~cm}^{-1}$. With threshold energy equal to $7105516 \mathrm{~cm}^{-1}$, we can find that energies of the $4 d^{9} 5 f^{2}$ level relative thresholds should be equal to 765000 $546000 \mathrm{~cm}^{-1}$. That differs substantially from the values $1068000-913000 \mathrm{~cm}^{-1}$ and $366000-218000 \mathrm{~cm}^{-1}$ obtained in the Dirac-Fock and the model-potential approximations. We hope to develop a new code that will allow us to incorporate the RMBPT approach in the evaluation of the autoionizing rates beyond the 'DF' or 'MP' approaches.

The comparison of the results obtained by different methods is very important when only a few experimental measurements are available. We conclude from the comparison of the results given in tables 2-4 that the energy values obtained by different codes are in good agreement $(0.1-$ $1 \%$ ), and the differences in the transition rates do not exceed $10-20 \%$ for largest values of those properties. Comparison of data in the cases where more accurate RMBPT codes are applicable give more confidence in data obtained in other systems where these methods cannot be used owing to complexity in the electronic structure. In table 1, we show configurations that should be used to produce the dielectronic satellite spectra and the (DR) rate coefficients of Ag-like tungsten from Pd-like tungsten. The use of the most complete set of states is very important to accurately evaluate the branching ratios. The summation over both non-autoionizing and autoionizing states has to be included. The version of the 
COWAN code [53] allows us to extract atomic properties in a more convenient way than the HULLAC code in order to evaluate the branching ratios and the effective emission rate coefficients of the dielectronic satellite lines. As a result, all numerical data and graphs below are obtained using COWAN code results. Since we use COWAN code values as our final results, it is essential to test this code on the cases where more accurate RMBPT methods work. This was the goal of the comparisons presented in tables 2-4.

\section{Dielectronic satellite spectra}

DR of the Pd-like $\mathrm{W}^{28+}$ ion proceeds via electron capture into the intermediate autoionizing states of the Ag-like $\mathrm{W}^{27+}$ ion followed by the radiative decay to singly excited bound states:

$$
\begin{aligned}
& \mathrm{W}^{28+}\left(4 \mathrm{~d}^{10}\right)+e \rightarrow \mathrm{W}^{27+*}\left(4 \mathrm{~d}^{9} n_{1} l_{1} n_{2} l_{2}\right) \\
& \quad \rightarrow \mathrm{W}^{27+*}\left(4 \mathrm{~d}^{10} n l+4 \mathrm{~d}^{9} 4 \mathrm{f} n l+4 \mathrm{~d}^{9} 5 l 5 l^{\prime}+4 \mathrm{~d}^{9} 5 \mathrm{~s} 6 l\right)+h v \\
& \quad \downarrow \\
& \quad \mathrm{W}^{28+}\left(4 \mathrm{~d}^{10}\right)+e .
\end{aligned}
$$

An alternative decay channel for the autoionizing level in equation (1) is via autoionization, and in this case the system returns to its original state $4 \mathrm{~d}^{10}$ as shown by a vertical arrow in equation (1).

Importantly, not all of the doubly excited states listed in the previous section can autoionize. The $4 \mathrm{~d}^{9} 4 \mathrm{f} 8 \mathrm{f}, 4 \mathrm{~d}^{9} 4 \mathrm{f} 9 l$, $4 \mathrm{~d}^{9} 5 \mathrm{~d} n l^{\prime} \quad(n=6-9), 4 \mathrm{~d}^{9} 5 \mathrm{f} n l(n=5-9), 4 \mathrm{~d}^{9} 5 \mathrm{gnl}(n=5-$ $9), 4 d^{9} 6 \operatorname{lnl}(n=6-7)$ levels are indeed autoionizing levels. However, the doubly excited $4 d^{9} 5 p 6 p, 4 d^{9} 5 d 5 f, 4 d^{9} 4 f 7 f$, $4 d^{9} 4 f 8 s, 4 d^{9} 4 f 8 p$ and $4 d^{9} 4 f 8 d$ configurations are only partly autoionizing, as some of the levels have energies below the ionization potential. Finally, none of the levels of the $4 \mathrm{~d}^{9} 4 \mathrm{f} n l$ $(n=4-7), 4 \mathrm{~d}^{9} 5 \mathrm{~s} 5 l, 4 \mathrm{~d}^{9} 5 \mathrm{p} 5 l, 4 \mathrm{~d}^{9} 5 \mathrm{~d}^{2}, 4 \mathrm{~d}^{9} 5 \mathrm{~s} 6 l$ and $4 \mathrm{~d}^{9} 5 \mathrm{p} 6 \mathrm{~s}$ configurations can autoionize.

During the radiative-stabilization phase of DR, dielectronic satellite lines are emitted when an electron jumps from a doubly excited autoionizing state to a singly and doubly excited bound state. For instance, the radiative transitions from the $4 \mathrm{~d}^{9} 5 \ln l^{\prime}$ and $4 \mathrm{~d}^{9} 6 \ln l^{\prime}$ states to the $4 \mathrm{~d}^{10} n l$ states give rise to satellite lines of the $4 d-5 l$, and $4 d-6 l$ lines of Pd-like tungsten. Assuming a Maxwellian distribution, the effective emission rate coefficient of a dielectronic satellite line can be written as (see, for example, [57])

$$
\begin{gathered}
C_{S}^{\mathrm{eff}}(j, i)=3.3 \times 10^{-24}\left(\frac{I_{H}}{k T_{e}}\right)^{3 / 2} \frac{Q_{d}(j, i)}{g_{0}} \\
\times \exp \left(-\frac{E_{S}(i)}{k T_{e}}\right) \text { photons } \mathrm{cm}^{3} \mathrm{~s}^{-1}
\end{gathered}
$$

where the intensity factor $Q_{d}(j, i)$ is defined as

$$
Q_{d}(j, i)=\frac{g(i) A_{a}\left(i, i_{0}\right) A_{r}(j, i)}{\sum_{i_{0}^{\prime}} A_{a}\left(i, i_{0}^{\prime}\right)+\sum_{k} A_{r}(k, i)},
$$

and $I_{H}$ is the ionization potential of hydrogen, $j$ is the lower bound state, $i$ is the upper autoionizing state, $i_{0}$ is the initial state (i.e. the ground state $4 \mathrm{~d}^{10}$ of the Pd-like ion) and $i_{0}^{\prime}$ is the possible final state for autoionization (again, only $4 \mathrm{~d}^{10}$ in this case). The statistical weight of the initial state $i_{0}$ is $g_{0}=1, g(i)$ is the statistical weight of the doubly excited state, $A_{a}\left(i, i_{0}\right)$ is the autoionization rate from $i$ to $i_{0}, A_{r}(j, i)$ is the radiative transition probability from $i$ to $j, E_{S}(i)$ is the excitation energy of the autoionizing state $i$ relative to the energy of the threshold and $T_{e}$ is the electron temperature. For some cases, $A_{a} \gg A_{r}$ and then $Q_{d}$ can be estimated as $Q_{d}(j, i) \approx g(i) A_{r}(j, i)$.

It follows from equation (2) that the intensity of dielectronic satellites depends on the intensity factor $Q_{d}(j, i)$ and excitation energy $\mathrm{E}_{S}(i)$. The strongest lines with $Q_{d}(j, i)>5.0 \times 10^{12} \mathrm{~s}^{-1}$ are presented in tables 5 and 6 for odd- and even-parity initial states, respectively. Since the sum over the final states of autoionization $i_{0}^{\prime}$ in equation (3) includes only one state, $4 \mathrm{~d}^{10}$, it is in fact reduced to a single rate $A_{a}(i)$. In addition to the $A_{a}(i)$ values, tables 5 and 6 also present $E_{S}(i)$, weighted radiative rates $g_{i} A_{r}(j, i)$, sums of weighted radiative rates $\sum_{k} g_{i} A_{r}(k, i)$, wavelengths $\lambda$ for dipole-allowed transitions, relative intensity factors $Q_{d}(j, i)$ and effective emission rate coefficients $C_{S}^{\text {eff }}(j, i)$. Short designations are used in these tables and in the text below: $4 \mathrm{~d}^{10} n l=n l, 4 \mathrm{~d}^{9} n \ln ^{\prime} l^{\prime}=n \ln ^{\prime} l^{\prime}$. The restriction $Q_{d}(j, i)>$ $5.0 \times 10^{12} \mathrm{~s}^{-1}$ leaves only a few dozen lines out of the total of $2.0 \times 10^{6}$ transitions, and most of these lines are due to the oneelectron $n l-4 \mathrm{~d}^{9} 5 \mathrm{f} n l, 4 \mathrm{~d}^{9} 4 \mathrm{f} n l-4 \mathrm{~d}^{9} 5 \mathrm{~d} n l$ and $4 \mathrm{~d}^{9} 4 \mathrm{f} n l-4 \mathrm{~d}^{9} 5 \mathrm{~g} n l$ transitions with $n=5-9$.

One can see from tables 5 and 6 that in $\mathrm{W}^{27+}$, in contrast to Na-like $\mathrm{W}^{63+}[14]$ and $\mathrm{Ag}$-like $\mathrm{Xe}^{7+}$ [46], there are a large number of transitions with non-autoionizing doubly excited lower states. In the case of Na-like $\mathrm{W}^{63+}$, only singly excited states are non-autoionizing, none of the doubly excited states are non-autoionizing. In the case of $\mathrm{Ag}$-like $\mathrm{Xe}^{7+}$, it was found that about 20 doubly excited states are non-autoionizing. In our case of Ag-like $\mathrm{W}^{27+}$, we found a very large number nonautoionizing doubly excited states (about 2000 states).

Strong mixing between some of doubly excited configurations was already noted in $[14,46]$. Strong mixing of some configurations also occurs in $\mathrm{W}^{27+}$. This effect is exemplified by the $4 \mathrm{~d}^{9} 4 \mathrm{f} 6 \mathrm{~d}^{2} I_{11 / 2}^{c}-4 \mathrm{~d}^{9} 5 \mathrm{f} 6 \mathrm{f}^{2} K_{13 / 2}^{c}$ transition in table 6 . The large intensity factor $Q_{d}$ for this transition is due to the mixing between the $4 d^{9} 5 f 6 f$ and $4 d^{9} 5 g 6 d$ configurations. However, a large value of $Q_{d}$ is per se insufficient to guarantee a large effective rate coefficient as the $\exp \left(-E_{s}(i) / k T_{e}\right)$ factor may strongly modify it. Indeed, the $C_{S}^{\text {eff }}$ coefficient for the $4 \mathrm{~d}^{10} 5 \mathrm{~d}^{2} \mathrm{D}_{5 / 2}-4 \mathrm{~d}^{9} 5 \mathrm{~d} 5 \mathrm{f}^{2} \mathrm{~F}_{7 / 2}^{e}$ transition (table 5) is larger than the $C_{S}^{\text {eff }} 4 \mathrm{~d}^{10} 5 \mathrm{~g}^{2} \mathrm{G}_{7 / 2}-4 \mathrm{~d}^{9} 5 \mathrm{f} 5 \mathrm{~g}{ }^{2} \mathrm{H}_{9 / 2}^{a}$ transition by a factor of 2.7, although the value of $Q_{d}$ for the former transition is smaller than that for the latter.

The strongest lines shown in the top right panel of figure 1 result from the $4 \mathrm{~d}^{10} 5 \mathrm{~d}^{2} \mathrm{D}_{5 / 2}-4 \mathrm{~d}^{9} 5 \mathrm{~d} 5 \mathrm{f}{ }^{2} \mathrm{P}_{3 / 2}^{c}$, $4 d^{10} 5 f^{2} \mathrm{~F}_{5 / 2}-4 d^{9} 5 f^{2}{ }^{2} \mathrm{~F}_{7 / 2}^{e}$ and $4 \mathrm{~d}^{10} 5 \mathrm{~d}^{2} \mathrm{D}_{5 / 2}-4 \mathrm{~d}^{9} 5 \mathrm{~d} 5 \mathrm{f}^{2} \mathrm{~F}_{5 / 2}$ transitions. Those three lines are the satellite lines to the strong $\lambda=20.594 \AA$ and $\lambda=21.106 \AA$ lines of the $4 \mathrm{~d}^{10}{ }^{1} \mathrm{~S}_{0}-4 \mathrm{~d}^{9} 5 \mathrm{f}^{1} \mathrm{P}_{1},{ }^{3} \mathrm{D}_{1}$ resonance transitions of Pd-like tungsten [58]. The satellite lines to another strong (4d-4f) line in Pd-like tungsten, located at $\lambda=48.948 \AA$ [59], are shown in the top right panel of figure 2 . The strongest satellite lines correspond to the $4 \mathrm{~d}^{10} 8 \mathrm{~s}^{2} \mathrm{~S}_{1 / 2}-4 \mathrm{~d}^{9} 4 \mathrm{f} 8 \mathrm{~s}{ }^{2} \mathrm{P}_{3 / 2}^{a}$ transition $(\lambda=49.119 \AA)$ and the $4 \mathrm{~d}^{10} 8 \mathrm{p}^{2} \mathrm{P}_{1 / 2}-4 \mathrm{~d}^{9} 4 \mathrm{f} 8 \mathrm{p}^{2} \mathrm{D}_{3 / 2}^{a}$ transition $(\lambda=49.121 \AA)$. The strongest lines shown in the 
Table 5. Autoionization rates $\left(A_{a}\right.$ in $\left.\mathrm{s}^{-1}\right)$ and excitation energies $\left(E_{S}\right.$ in $\left.\mathrm{eV}\right)$ for the $4 \mathrm{~d}^{9} 4 \mathrm{f} n l^{\prime}$ and $4 \mathrm{~d}^{9} 5 \ln l^{\prime}$ and $4 \mathrm{~d}^{9} 6 \mathrm{f} n l^{\prime}$ levels. Wavelengths $\left(\lambda\right.$ in $\AA$ ), weighted radiative rates $\left(g A_{r}\right.$ in s $\left.{ }^{-1}\right)$, intensity factors $\left(Q_{d}\right.$ in s$\left.{ }^{-1}\right)$ and effective emission rate coefficients $\left(C_{S}^{\text {eff }}\right.$ in $\left.\mathrm{cm}^{3} \mathrm{~s}^{-1}\right)$ for transitions between the $4 \mathrm{~d}^{10} \mathrm{nl}$ and $4 \mathrm{~d}^{9} 4 \mathrm{f} 5 l^{\prime}$ even-parity excited and the $4 \mathrm{~d}^{9} n l n^{\prime} l^{\prime}$ odd-parity autoionization levels of Ag-like tungsten. The $C_{S}^{\text {eff }}$ values are given for $T_{\mathrm{e}}=100 \mathrm{eV}$. Designations: $4 \mathrm{~d}^{10} n l=n l, 4 \mathrm{~d}^{9} n l n^{\prime} l^{\prime}=n n^{\prime} l^{\prime}$. The upper indices are used in the Cowan code to differentiate between atomic terms. $\mathrm{A}[\mathrm{B}]$ means $\mathrm{A} \cdot 10^{B}$.

\begin{tabular}{|c|c|c|c|c|c|c|c|c|}
\hline $\begin{array}{l}\text { Low level } \\
\left({ }^{1} \mathrm{~S}\right) n l L \mathrm{~S} J\end{array}$ & $\begin{array}{l}\text { Upper level } \\
n \ln ^{\prime} l^{\prime} L S J\end{array}$ & $\begin{array}{l}A_{a} \\
\mathrm{~s}^{-1}\end{array}$ & $\begin{array}{l}E_{S} \\
\mathrm{eV}\end{array}$ & $\begin{array}{l}\sum g A_{r} \\
\mathrm{~s}^{-1}\end{array}$ & $\begin{array}{l}g A_{r} \\
\mathrm{~s}^{-1}\end{array}$ & $\begin{array}{l}\lambda \\
\AA\end{array}$ & $\begin{array}{c}Q_{d} \\
\mathrm{~s}^{-1}\end{array}$ & $\begin{array}{c}C_{S}^{\mathrm{eff}} \\
\mathrm{cm}^{3} \mathrm{~s}^{-1}\end{array}$ \\
\hline$/ 2$ & 0 & [12] & 50 & $72[12]$ & $7.29[12]$ & 15.88 & $6.30[12]$ & $6.42[-15]$ \\
\hline$d^{2} D_{5 / 2}$ & $6 \mathrm{f} 7 \mathrm{~d}^{2} \mathrm{~F}_{7 / 2}^{a}$ & $1.36[12]$ & 491.92 & $1.47[13]$ & $1.41[13]$ & 16.24 & $98[12]$ & $7.23[-15]$ \\
\hline$d^{2} D_{5 / 2}$ & $6 \mathrm{f} 7 \mathrm{~d}^{2} \mathrm{~F}_{7 / 2}^{a}$ & $1.36[12]$ & 491.92 & $1.47[13]$ & $1.41[13]$ & 16.24 & $5.98[12]$ & $7.23[-15]$ \\
\hline $\mathrm{g}^{2} \mathrm{G}_{9 / 2}$ & $6 \mathrm{f} 7 \mathrm{~g}^{2} \mathrm{H}_{11 / 2}^{a}$ & $5.69[11]$ & 536.59 & 3.89 [13] & $3.69[13]$ & 16.24 & $5.51[12]$ & $4.26[-15]$ \\
\hline$d^{2} D_{3 / 2}$ & $6 \mathrm{f} 7 \mathrm{~d}^{4} \mathrm{P}_{5 / 2}^{a}$ & $1.72[12]$ & 490.15 & $8.66[12]$ & $7.60[12]$ & 16.24 & $4.13[12]$ & $5.08[-15]$ \\
\hline$d^{2} D_{5 / 2}$ & ${ }^{2} \mathrm{~F}_{7 / 2}^{e}$ & $9.38[12]$ & 3.38 & $1.54[13]$ & $1.24[13]$ & 20.73 & $1.03[13]$ & $1.65[-12]$ \\
\hline $\mathrm{g}^{2} \mathrm{G}_{7 / 2}$ & $5 \mathrm{~g}^{2} \mathrm{H}_{9 / 2}^{a}$ & $3.25[12]$ & 152.49 & $3.95[13]$ & $3.78[13]$ & 20.80 & $1.71[13]$ & $6.16[-13]$ \\
\hline $5 \mathrm{~g}^{2} \mathrm{G}_{7 / 2}$ & $5 f 5 g^{2} \mathrm{H}_{9 / 2}^{f}$ & $2.92[13]$ & 145 & $3.64[13]$ & $2.27[13]$ & 21.04 & 2.02 & $7.79[-13]$ \\
\hline $4 \mathrm{f} 5 \mathrm{p}^{4} \mathrm{H}_{7 / 2}^{b}$ & 5 p6g ${ }^{4} I_{9 / 2}^{a}$ & & & & $2.41[13]$ & 21.11 & $1.33[13]$ & $5.88[-13]$ \\
\hline $6 \mathrm{~g}^{2} \mathrm{G}_{9 / 2}$ & $5 f 6 g^{2} \mathrm{H}_{11 / 2}^{a}$ & $2.78[12]$ & 278.11 & $8.00[13]$ & $4.56[13]$ & 21.12 & $1.34[13]$ & $1.37[-13]$ \\
\hline $6 d^{2} D_{5 / 2}$ & $5 \mathrm{f}^{2} \mathrm{~d}^{2} \mathrm{~F}_{7 / 2}^{a}$ & $3.63[12]$ & 201.58 & $1.84[13]$ & $1.80[13]$ & 21.13 & $1.10[13]$ & $2.43[-13]$ \\
\hline $4 f 5 p^{2} G_{7 / 2}^{c}$ & $5 \mathrm{p} 6 \mathrm{~g}{ }^{2} \mathrm{H}_{9 / 2}^{a}$ & $7.06[12]$ & 116.62 & 3.10 [13] & 2.28 [13] & 21.14 & 1.59 [13] & $8.20[-13]$ \\
\hline $6{ }^{2} \mathrm{G}_{9 / 2}$ & $5 f 6 g^{2} \mathrm{G}_{9 / 2}^{a / 2}$ & & & & & & & $1.16[-13]$ \\
\hline $4 \mathrm{f} 5 \mathrm{p}^{2} \mathrm{G}_{9 / 2}^{f}$ & $5 \mathrm{p} 6 \mathrm{~g}{ }^{2} \mathrm{H}_{11 / 2}^{a}$ & $3.47[12]$ & 116.72 & 4.4 & 2.79 & 21.24 & & {$[-13]$} \\
\hline $4 \mathrm{f} 5 \mathrm{p}^{4} \mathrm{H}_{9 / 2}^{a / 2}$ & $5 \mathrm{p} 6 \mathrm{~g}{ }^{2} \mathrm{H}_{11 / 2}^{a}$ & $3.47[12]$ & 104.87 & $4.46[13]$ & $2.15[13]$ & 21.38 & $1.04[13]$ & $6.03[-13]$ \\
\hline $4 \mathrm{f} 5 \mathrm{f}^{2} \mathrm{~K}_{13 / 2}^{c / 2}$ & $5 f 5 g^{2} L_{15 / 2}^{c}$ & $1.50[12]$ & 165.27 & $9.01[13]$ & $7.77[13]$ & 27.59 & $1.64[13]$ & $5.20[-13]$ \\
\hline $4 \mathrm{f} 5 \mathrm{f}^{2} \mathrm{H}_{11 / 2}^{e}$ & $5 \mathrm{f}^{2} \mathrm{~g}^{2} \mathrm{~K}_{13 / 2}^{d / 2}$ & $2.53[12]$ & 148.43 & $7.43[13]$ & $5.66[13]$ & 27.60 & $1.83[13]$ & $6.87[-13]$ \\
\hline $4 \mathrm{f} 5 \mathrm{f}^{2} \mathrm{I}_{11 / 2}^{e}$ & $5 f 5 g^{2} K_{13 / 2}^{d}$ & $2.53[12]$ & 164.80 & $7.43[13]$ & $5.94[13]$ & 27.61 & $1.92[13]$ & $6.12[-13]$ \\
\hline $4 \mathrm{f} 5 \mathrm{f}^{2} \mathrm{H}_{9 / 2}^{e}$ & $5 \mathrm{f}^{8} \mathrm{~g}^{2} \mathrm{I}_{11 / 2}^{e}$ & $1.07[13]$ & 147.48 & $6.88[13]$ & $2.26[13]$ & 27.69 & $1.47[13]$ & $5.57[-13]$ \\
\hline $8 d^{2} D_{3 / 2}$ & $\mathrm{P}_{1 / 2}^{a}$ & $7.41[12]$ & 49.44 & $1.22[13]$ & 1.10 [13] & 49.12 & $6.06[12]$ & $6.12[-13]$ \\
\hline $8 s^{2} S_{1 / 2}$ & $4 \mathrm{f} 8 \mathrm{~s}{ }^{2} \mathrm{P}_{1 / 2}^{a}$ & $1.30[13]$ & 22.92 & $1.26[13]$ & $1.04[13]$ & 49.12 & $7.04[12]$ & $9.26[-13]$ \\
\hline $8 \mathrm{~s}^{2} \mathrm{~S}_{1 / 2}$ & $4 \mathrm{f} 8 \mathrm{~s}^{2} \mathrm{P}_{3 / 2}^{a / 2}$ & $1.31[13]$ & 22.92 & $2.45[13]$ & $2.09[13]$ & 49.12 & $1.42[13]$ & $1.87[-12]$ \\
\hline $9 \mathrm{~s}^{2} \mathrm{~S}_{1 / 2}$ & $4 \mathrm{f} 9 \mathrm{~s}^{2} \mathrm{P}_{1 / 2}^{a / 2}$ & $1.12[13]$ & 77.61 & $1.17[13]$ & $1.03[13]$ & 49.13 & $6.79[12]$ & $5.17[-13]$ \\
\hline $9 \mathrm{~s}^{2} \mathrm{~S}_{1 / 2}$ & $4 \mathrm{f} 9 \mathrm{~s}{ }^{2} \mathrm{P}_{3 / 2}^{a}$ & $1.16[13]$ & 77.61 & $2.30[13]$ & $1.95[13]$ & 49.13 & $1.31[13]$ & $9.98[-13]$ \\
\hline $9 d^{2} D_{3 / 2}$ & $4 \mathrm{f} 9 \mathrm{~d}^{2} \mathrm{P}_{1 / 2}^{a}$ & $6.70[12]$ & 95.39 & $1.11[13]$ & $1.07[13]$ & 49.20 & $5.84[12]$ & $3.72[-13]$ \\
\hline
\end{tabular}

bottom right panel of figure 1 result from the transitions between the non-autoionizing $4 \mathrm{~d}^{9} 4 \mathrm{f} 5 \mathrm{~d}$ doubly excited states and the autoionizing $4 \mathrm{~d}^{9} 5 \mathrm{~d} 5 \mathrm{~g}$ doubly excited states. The wavelengths of the five $4 \mathrm{~d}^{9} 4 \mathrm{f} 5 \mathrm{~d}^{2,4} L_{J}-4 \mathrm{~d}^{9} 5 \mathrm{~d} 5 g{ }^{2,4} L_{J^{\prime}}^{\prime}$ lines are in the narrow range of $\lambda=27.67-27.95 \AA$. The values of the effective emission rate coefficient $C_{S}^{\text {eff }}\left(4 \mathrm{~d}^{9} 4 \mathrm{f} 5 \mathrm{~d}^{2,4} L_{J}-\right.$ $\left.4 \mathrm{~d}^{9} 5 \mathrm{~d} 5 \mathrm{~g}^{2,4} L_{J^{\prime}}^{\prime}\right)$ are about $105-208$ in units of $10^{-14} \mathrm{~cm}^{3} \mathrm{~s}^{-1}$. The $4 \mathrm{~d}^{9} 4 \mathrm{f} 5 \mathrm{~s}^{2} H_{9 / 2}^{b}-4 \mathrm{~d}^{9} 5 \mathrm{~s} 6 g^{2} I_{11 / 2}^{a}$ transition between doubly excited levels leads to the strong line shown in the top right panel of figure $1\left(\lambda=21.157 \AA\right.$ and $C_{S}^{\text {eff }}=1.05 \times 10^{-12}$ $\mathrm{cm}^{3} \mathrm{~s}^{-1}$ ).

The strongest lines shown on the left top panel of figure 1 are due to the Rydberg transitions $4 d^{10} 4 f-4 d^{9} 4 f 9 f$, $4 \mathrm{~d}^{10} 4 \mathrm{f}-4 \mathrm{~d}^{9} 4 \mathrm{f} 8 \mathrm{f}, 4 \mathrm{~d}^{10} 4 \mathrm{f}-4 \mathrm{~d}^{9} 4 \mathrm{f} 7 \mathrm{f}$ (in the range of $\lambda=13-$ $14 \AA), 4 \mathrm{~d}^{10} 5 \mathrm{p}-4 \mathrm{~d}^{9} 5 \mathrm{p} 6 \mathrm{p}, 4 \mathrm{~d}^{10} 5 \mathrm{p}-4 \mathrm{~d}^{9} 5 \mathrm{p} 6 \mathrm{f}, 4 \mathrm{~d}^{10} 5 \mathrm{p}-4 \mathrm{~d}^{9} 5 \mathrm{p} 7 \mathrm{f}$, $4 d^{10} 5 \mathrm{~s}-4 d^{9} 5 \mathrm{~s} 6 \mathrm{f}, 4 \mathrm{~d}^{10} 5 \mathrm{~d}-4 \mathrm{~d}^{9} 5 \mathrm{~d} 6 \mathrm{p}$ and $4 \mathrm{~d}^{10} 5 \mathrm{p}-4 \mathrm{~d}^{9} 5 \mathrm{p} 6 \mathrm{f}$ (in the range of $\lambda=16-18 \AA$ ). Other transitions between singly and doubly excited states are illustrated in the left panel of figure 2. The strongest features in this panel are due to the $4 d^{10} 6 p-4 d^{9} 5 p 6 p, 4 d^{10} 6 d-4 d^{9} 5 p 6 d$ and $4 d^{10} 6 g-4 d^{9} 5 p 6 g$ transitions in the narrow region around 29.0-29.1.

The strongest lines shown in the left bottom panel of figure 1 are due to $4 d^{9} 4 f 5 s-4 d^{9} 5 s 6 g$ transitions, which involve only doubly excited states. The values of the effective emission rate coefficient $C_{S}^{\text {eff }}$ are about $10.1-24.5$ in units of $10^{-14}$ $\mathrm{cm}^{3} \mathrm{~s}^{-1}$, and the wavelength region is 22.6-23.2 $\AA$. Other transitions between doubly excited states are illustrated by the bottom panels of figure 2. On the left bottom panel of figure 2 , the strongest lines are due to $4 d^{9} 5 \mathrm{~s} 5 \mathrm{f}-4 \mathrm{~d}^{9} 5 \mathrm{~s} 6 \mathrm{~g}$ transitions $(\lambda=$ 61.1-62.7 $\AA$ ) and the $4 d^{9} 5 d^{2}-4 d^{9} 5 d 6 p$ transitions $(\lambda=70.5-$ $71.1 \AA)$. Finally, the strongest lines, shown in the right bottom panel of figure 2 are due to $4 d^{9} 5 d^{2}-4 d^{9} 5 d 5 f$ transitions $(\lambda=$ $129-142 \AA)$, the $4 d^{9} 5 d 5 f-4 d^{9} 5 f^{2}$ transitions $(\lambda=140-156 \AA)$ and $4 d^{9} 5 \mathrm{p} 5 \mathrm{f}-4 \mathrm{~d}^{9} 5 \mathrm{~d} 5 \mathrm{f}$ transitions $(\lambda=159-163 \AA)$.

\section{Dielectronic recombination rate coefficients for excited states}

The DR rate coefficients for excited states are obtained by summation of the effective emission rate coefficients $C_{S}^{\text {eff }}(j, i)$ (equation (2)) for DR processes through all possible intermediate doubly excited states:

$$
\alpha_{d}\left(i_{0}, j\right)=\sum_{i} C_{S}^{\mathrm{eff}}(j, i) .
$$

For the DR process described by equation (1), one has to calculate $\alpha_{d}\left(i_{0}, j\right)$ with $i_{0}=4 \mathrm{~d}^{10}$ and all possible autoionizing doubly excited states $i$ of $\mathrm{W}^{27+}$ with energies larger than the $4 \mathrm{~d}^{10}$ threshold $I_{\text {th }}=7109000 \mathrm{~cm}^{-1}$ [24]. Among the doubly excited $4 \mathrm{~d}^{9} 4 \mathrm{f} n l$ and $4 \mathrm{~d}^{9} 5 \ln l^{\prime}(n=5-9)$ states, 5905 states of even parity and 6009 states of odd parity have energies above $I_{\text {th }}$. The doubly excited $4 \mathrm{~d}^{9} 6 \ln l^{\prime}(n=6-7)$ configurations 
Table 6. Autoionization rates $\left(A_{a}\right.$ in s $\left.{ }^{-1}\right)$ and excitation energies $\left(E_{S}\right.$ in $\left.\mathrm{eV}\right)$ for the $4 \mathrm{~d}^{9} 4 \mathrm{f} n l^{\prime}, 4 \mathrm{~d}^{9} 5 \ln l^{\prime}$ and $4 \mathrm{~d}^{9} 6 \mathrm{f} n l^{\prime}$ levels. Wavelengths $\left(\lambda\right.$ in $\AA$ ), weighted radiative rates $\left(g A_{r}\right.$ in s $\left.{ }^{-1}\right)$, intensity factors $\left(Q_{d}\right.$ in s $\left.{ }^{-1}\right)$ and effective emission rate coefficients $\left(C_{S}^{\text {eff }}\right.$ in $\left.\mathrm{cm}^{3} \mathrm{~s}^{-1}\right)$ for transitions between the $4 \mathrm{~d}^{10} n l$ and $4 \mathrm{~d}^{9} 4 \mathrm{f} n l^{\prime}$ even-parity excited and the $4 \mathrm{~d}^{9} n l n^{\prime} l^{\prime}$ odd-parity autoionization states of Ag-like tungsten. The $C_{S}^{\text {eff }}$ values are given for $T_{\mathrm{e}}=100 \mathrm{eV}$. Designations: $4 \mathrm{~d}^{10} n l=n l, 4 \mathrm{~d}^{9} n l n^{\prime} l^{\prime}=n n^{\prime} l^{\prime}$. The upper indices are used in the Cowan code to differentiate between atomic terms. $\mathrm{A}[\mathrm{B}]$ means $\mathrm{A} \cdot 10^{B}$.

\begin{tabular}{|c|c|c|c|c|c|c|c|c|}
\hline $\begin{array}{l}\text { Low level } \\
\left({ }^{1} \mathrm{~S}\right) n l L S J\end{array}$ & $\begin{array}{l}\text { Upper level } \\
n l n^{\prime} l^{\prime} L S J\end{array}$ & $\begin{array}{c}A_{a} \\
\left(\mathrm{~s}^{-1}\right)\end{array}$ & $\begin{array}{c}E_{S} \\
(\mathrm{eV})\end{array}$ & $\begin{array}{c}\sum \mathrm{gA}_{r} \\
\left(\mathrm{~s}^{-1}\right)\end{array}$ & $\begin{array}{l}\mathrm{gA}_{r} \\
\left(\mathrm{~s}^{-1}\right)\end{array}$ & $\begin{array}{l}\lambda \\
\AA\end{array}$ & $\begin{array}{c}\mathrm{Q}_{d} \\
\left(\mathrm{~s}^{-1}\right)\end{array}$ & $\begin{array}{c}\mathrm{C}_{S}^{\mathrm{eff}} \\
\left(\mathrm{cm}^{3} \mathrm{~s}^{-1}\right)\end{array}$ \\
\hline & 2 & & & & $382[12]$ & - & $251[12]$ & -1 \\
\hline${ }^{2} \mathrm{~F}_{7 / 2}$ & $3 f^{2} G_{9 / 2}^{c}$ & $34[12]$ & 21.19 & $17[12]$ & $59[12]$ & .79 & . $.04[12]$ & $1.8 /[-13]$ \\
\hline${ }^{2} \mathrm{~F}_{5 / 2}$ & $7 f^{2} G_{7 / 2}^{g}$ & $77[12]$ & 517.80 & $73[13]$ & 10 [13] & 6.22 & $.94[12]$ & $61[-15]$ \\
\hline${ }^{2} \mathrm{P}_{3 / 2}$ & $6 f 7 p^{2} \mathrm{~F}_{5 / 2}^{d^{\prime}}$ & $23[12]$ & 70.03 & $32[13]$ & & 6.23 & $.30[12]$ & $1.25[-14]$ \\
\hline $4 f 5 d^{2} F_{5 / 2}^{j}$ & $5 \mathrm{~d} 9 \mathrm{~g}^{2} \mathrm{G}_{7 / 2}^{a}$ & 2 & 34 & 2] & 4.5 & 16.25 & 4.3 & $43[\quad 14]$ \\
\hline${ }^{4} \mathrm{D}_{5 / 2}^{d}$ & $5 \mathrm{~d} 9 \mathrm{~g}^{2} \mathrm{G}_{7 / 2}^{g}$ & $.74[11]$ & 17 & 2] & 4.31 & 16.40 & 2] & $27[-15]$ \\
\hline $5 d^{2} F_{5 / 2}^{j}$ & $5 \mathrm{~d} 8 \mathrm{~g}^{2} \mathrm{G}_{7 / 2}^{a}$ & $2.72[13]$ & 355.11 & 8.35 & 6.72 & 17.08 & 6.48 & $.08[-14]$ \\
\hline $\mathrm{G}_{7 / 2}^{d}$ & $5 \mathrm{~d} 8 \mathrm{~g}{ }^{4} \mathrm{~F}_{9 / 2}^{a}$ & $1.12[12]$ & 345.02 & 3] & 7.13 & 17.20 & 2] & $87[-14]$ \\
\hline $5 \mathrm{~d}^{2} \mathrm{~F}_{7 / 2}^{i / 2}$ & $5 \mathrm{~d} 7 \mathrm{~g}^{2} \mathrm{G}_{9 / 2}^{a}$ & 682 & 3 & & 1.6 & & & $0[-13]$ \\
\hline$p^{2} \mathrm{P}_{1 / 2}$ & $5 \mathrm{f} 6 \mathrm{p}^{2} \mathrm{D}_{3 / 2}^{a}$ & 1 & 1 & 2.2 & 2.06 & .61 & 3] & $-13]$ \\
\hline $8 \mathrm{p}^{2} \mathrm{P}_{1 / 2}^{1 / 2}$ & $5 \mathrm{f} 8 \mathrm{p}^{2} \mathrm{D}_{3 / 2}^{a}$ & $9.06[11]$ & 367.60 & $2.39[13]$ & $2.34[13]$ & 21.04 & $3.07[12]$ & $1.29[-14]$ \\
\hline $4 \mathrm{f} 5 \mathrm{~d}^{4} \mathrm{G}_{5 / 2}^{d}$ & $5 \mathrm{~d} 6 \mathrm{~g}{ }^{4} \mathrm{H}_{7 / 2}^{a}$ & $2.14[13]$ & 207.04 & $2.41[13]$ & $1.5 \mathrm{c}$ & 21.11 & 3] & $2.90[-13]$ \\
\hline $4 \mathrm{f} 5 \mathrm{~s}^{2} \mathrm{H}_{9 / 2}^{b}$ & $5 \mathrm{~s} 6 \mathrm{~g}^{2} I_{11 / 2}^{a}$ & 1. & & & & 16 & & $1.02[-12]$ \\
\hline $5 \mathrm{f}^{2} \mathrm{~F}_{7 / 2}$ & $5 \mathrm{f}^{2}{ }^{2} \mathrm{D}_{5 / 2}$ & $6.74[12]$ & 75.11 & 1.71 & 1.6 & 21.19 & 1.18 [13] & $9.22[-13]$ \\
\hline $8 \mathrm{~s}{ }^{4} \mathrm{H}_{11 / 2}$ & & & & & & & & $06[-14]$ \\
\hline $6 s{ }^{4} \mathrm{H}_{9 / 2}$ & $5 \mathrm{~g} 6 \mathrm{~s}^{2} \mathrm{I}_{11 / 2}^{a}$ & & 19 & & & 27 & & $4.23[-13]$ \\
\hline $6 \mathrm{~d}^{2} \mathrm{I}_{11 / 2}^{c^{c}}$ & $5 \mathrm{f} 6 \mathrm{f}^{2} \mathrm{~K}_{13 / 2}^{c}$ & $5.25[11]$ & 286.85 & $5.99[12]$ & 3.86 & 27.43 & $2.13[13]$ & $2.00[-13]$ \\
\hline $5 \mathrm{~d}^{4} \mathbf{I}_{9 / 2}^{b}$ & $5 \mathrm{~d} 5 g{ }^{4} \mathrm{~K}_{11 / 2}^{b}$ & & & & & & & $9.59[-13]$ \\
\hline $6 d^{2} I_{13 / 2}^{a}$ & $5 \mathrm{~g} 6 \mathrm{~d}^{2} \mathrm{~K}_{15 / 2}^{a}$ & $7.48[11]$ & 28 & 8.3 & & & & $9.89[-14]$ \\
\hline $5 d^{4} G_{5 / 2}^{d}$ & $5 \mathrm{~d} 5 g{ }^{4} \mathrm{H}_{7 / 2}^{a}$ & & 59.28 & $4.22[13]$ & & 27.67 & & $1.58[-12]$ \\
\hline $5 \mathrm{~d}^{2} \mathrm{~F}_{7 / 2}^{i}$ & $5 \mathrm{~d} 5 \mathrm{~g}^{2} \mathrm{G}_{9 / 2}^{a}$ & $1.43[14]$ & 80.02 & $3.74[13]$ & $3.15[1$ & 27.77 & $3.07[13]$ & $2.28[-12]$ \\
\hline $4 \mathrm{f} 5 \mathrm{~d}^{2} \mathrm{~F}_{7 / 2}^{i}$ & $5 \mathrm{~d} 5 \mathrm{~g}{ }^{4} \mathrm{G}_{9 / 2}^{b}$ & & & & & & & $1.09[-12]$ \\
\hline $\mathrm{f} 5 \mathrm{~d}^{4} I_{11 / 2}^{a}$ & $5 \mathrm{~d} 5 g^{2} I_{13 / 2}^{a}$ & $2.62[12]$ & 67.74 & & & 27.91 & $2.14[13]$ & $1.80[-12]$ \\
\hline & $4 \mathrm{f} 8 \mathrm{p}^{2} \mathrm{~S}_{1 / 2}^{a}$ & $3.60[12]$ & 36.05 & & & 49.07 & $4.07[12]$ & $4.70[-13]$ \\
\hline $8 \mathrm{p}^{2} \mathrm{P}_{1 / 2}$ & $4 \mathrm{f} 8 \mathrm{p}^{2} \mathrm{D}_{3 / 2}^{a}$ & $3.41[12]$ & 30.93 & $2.45[13]$ & $2.32[13]$ & 49.12 & $8.28[12]$ & $1.01[-12]$ \\
\hline
\end{tabular}

include 1205 levels of even parity and 1043 levels of odd parity.

The sum over $i$ in equation (4) includes the $4 \mathrm{~d}^{9} 4 \mathrm{f} n_{1} l$, $4 \mathrm{~d}^{9} 5 \ln _{1} l^{\prime}$ and $4 \mathrm{~d}^{9} 6 \ln _{2} l^{\prime}$ doubly excited, autoionizing states with $n_{1}=5-9$ (11 914 levels) and $n_{2}=6-7$ (2248 levels). In figure 3 , we illustrate the contributions of the $4 \mathrm{~d}^{9} 4 \mathrm{f} n l$ ( $n=$ $7-9), 4 \mathrm{~d}^{9} 5 l^{\prime} n l(n=5-9)$ and $4 \mathrm{~d}^{9} 6 l^{\prime} n l(n=6-7)$ states to the DR rate coefficients $\alpha_{d}\left(4 \mathrm{~d}^{10}, j\right)$ for the $j=4 \mathrm{~d}^{10} n l^{2} L_{J}$ states as a function of $T_{e}$ in $\mathrm{Ag}$-like tungsten. It should be noted that we use an abbreviated notation to label the curves in figure 3 : $4 \mathrm{~d} n l^{\prime} n^{\prime \prime}$ instead of $4 \mathrm{~d}^{9} n l^{\prime} n^{\prime \prime}$ and $n l$ instead of $4 \mathrm{~d}^{10} n l$.

In order to estimate the contributions from the high- $n$ autoionizing states to the DR rate coefficients associated with excited states, i.e. sum over $i$ with $n>9$ for $4 \mathrm{~d}^{9} 4 \mathrm{f} n l^{\prime}$ and $4 \mathrm{~d}^{9} 5 l^{\prime} n l$ autoionizing states and with $n>7$ for the $4 \mathrm{~d}^{9} 6 l^{\prime} n l$ autoionizing states, we use empirical scaling laws [36], which can only be implemented to include one-electron $4 \mathrm{~d}-n \mathrm{p}$, and $4 \mathrm{~d}-n \mathrm{f}$ dipole transitions. The contributions from the high- $n$ states appear for the first low-lying configurations $4 \mathrm{~d}^{10} 4 \mathrm{f}$ and $4 \mathrm{~d}^{10} n l$. For these configurations, the $4 \mathrm{f}-4 \mathrm{~d} 4 \mathrm{f} n \mathrm{p}, 4 \mathrm{f}-4 \mathrm{~d} 4 \mathrm{f} n \mathrm{f}$, $5 l-4 \mathrm{~d} 5 \ln \mathrm{p}, 5 l-4 \mathrm{~d} 5 \ln \mathrm{f}, 6 l-4 \mathrm{~d} 6 \ln \mathrm{p}$, and $6 l-4 \mathrm{~d} 6 \ln \mathrm{f}$ transitions with $n>9$ and $l=\mathrm{s}, \mathrm{p}, \mathrm{d}, \mathrm{f}, \mathrm{g}$ need to be included as well.

To estimate $Q_{d}(j, i)$ in equation (3) for autoionization states $i$ with high principal quantum number $n$ for the $4 \mathrm{~d} 4 \mathrm{f} n l$, $4 \mathrm{~d} 5 l^{\prime} n l$, and $4 \mathrm{~d} 6 l^{\prime} n l$ configurations and for the $4 \mathrm{~d}-n \mathrm{p}$ and $4 \mathrm{~d}-n \mathrm{f}$ dipole transitions, we used our calculated data for $n=$
9 and the $1 / n^{3}$ scaling law for rates $A_{a}$ and $A_{r}$. For example, the formulae for the $5 \mathrm{~d}-4 \mathrm{~d} 5 \mathrm{~d} n \mathrm{f}$ case are

$$
A_{a}\left(4 \mathrm{~d} 5 \mathrm{~d} n \mathrm{f}^{2,4} L_{J}\right)=\left(\frac{9}{n}\right)^{3} A_{a}\left(4 \mathrm{~d} 5 \mathrm{~d} 9 \mathrm{f}^{2,4} L_{J}\right),
$$

$$
\begin{aligned}
A_{r} & \left(5 \mathrm{~d}^{2} \mathrm{D}_{3 / 2}-4 \mathrm{~d} 5 \mathrm{~d} n \mathrm{f}^{2,4} L_{J}\right) \\
= & \left(\frac{9}{n}\right)^{3} A_{r}\left(5 \mathrm{~d}^{2} \mathrm{D}_{3 / 2}-4 \mathrm{~d} 5 \mathrm{~d} 9 \mathrm{f}^{2,4} L_{J}\right) \\
& \times\left(\frac{\Delta E\left(5 \mathrm{~d}^{2} \mathrm{D}_{3 / 2}-4 \mathrm{~d} 5 \mathrm{~d} n \mathrm{f}^{2,4} L_{J}\right)}{\Delta E\left(5 \mathrm{~d}^{2} \mathrm{D}_{3 / 2}-4 \mathrm{~d} 5 \mathrm{~d} 9 \mathrm{f}^{2,4} L_{J}\right)}\right)^{3}
\end{aligned}
$$

In order to obtain the energies of the $4 \mathrm{~d} 5 \mathrm{~d} n \mathrm{f}^{2,4} L_{J}$ states as a function of $n$, the following asymptotic formula is proposed (see $[28,60])$ :

$$
E(4 \mathrm{~d} 5 \mathrm{~d} n \mathrm{f})-E(4 \mathrm{~d} 5 \mathrm{~d})=-\frac{1}{2 n^{2}}\left(Z-35+\frac{b(l)}{n}\right)^{2},
$$

where $b(l)$ is the $l$-dependent screening constant. Consequently, the energy differences in Ag-like W $(Z=74)$ can be found using the following formula:

$$
\begin{aligned}
& \Delta E\left(5 \mathrm{~d}^{2} \mathrm{D}_{3 / 2}-4 \mathrm{~d} 5 \mathrm{~d} n \mathrm{f}^{2,4} L_{J}\right) \\
& =\Delta E\left(5 \mathrm{~d}^{2} \mathrm{D}_{3 / 2}-4 \mathrm{~d} 5 \mathrm{~d} 9 \mathrm{f}^{2,4} L_{J}\right)-\frac{1521}{2}\left(\frac{1}{n^{2}}-\frac{1}{9^{2}}\right) .
\end{aligned}
$$



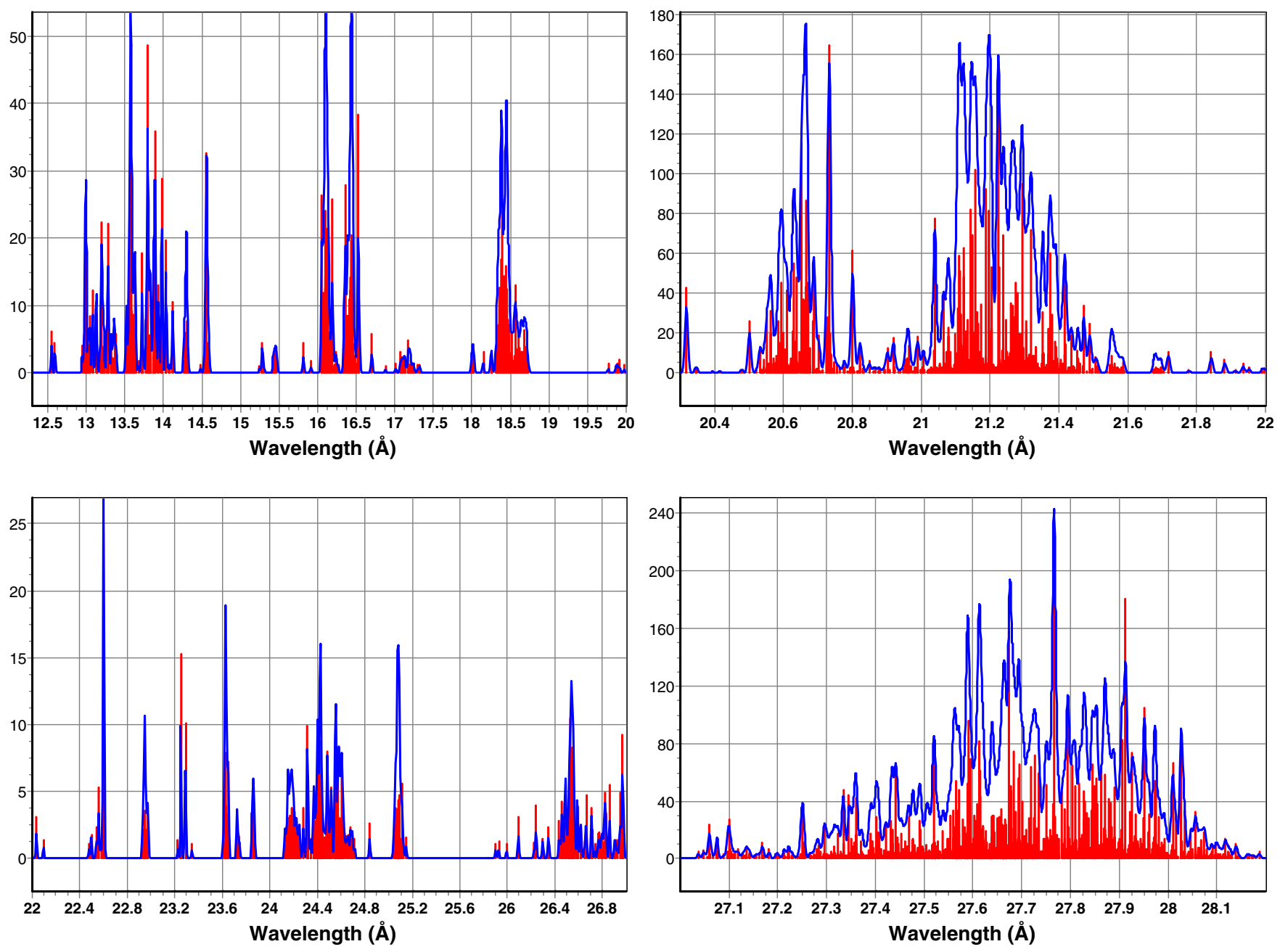

Figure 1. Synthetic spectra (red) of dielectronic satellite lines $\left(\left[4 \mathrm{~d}^{10} n l+4 \mathrm{~d}^{9} 4 \mathrm{f} n l+4 \mathrm{~d}^{9} 5 l 5 l^{\prime}+4 \mathrm{~d}^{9} 5 \mathrm{~s} 6 l\right]-4 \mathrm{~d}^{9} n_{1} l_{1} n_{2} l_{2}\right)$ from the $\mathrm{W}^{27+}$ ion at $T_{e}=100 \mathrm{eV}$ for $\lambda=12-28 \AA$. A resolving power, $R=\lambda / \Delta \lambda=1000$ (top left panel), 2000 (top right and bottom left panels) and 4000 (bottom right panel) is assumed to produce a Gaussian profile (blue). The scale in the ordinate is in units of $10^{-14} \mathrm{~cm}^{3} \mathrm{~s}^{-1}$.

A similar formula was used for the excitation energies $E_{S}(i)$ in equation (4) for $i=4 \mathrm{~d} 5 \mathrm{~d} n \mathrm{f}^{2,4} L_{J}$ :

$$
\begin{aligned}
& E_{S}\left(4 \mathrm{~d} 5 \mathrm{~d} n \mathrm{f}^{2,4} L_{J}\right)=E_{S}\left(4 \mathrm{~d} 5 \mathrm{~d} 9 \mathrm{f}^{2,4} L_{J}\right) \\
& -\frac{1521}{2}\left(\frac{1}{n^{2}}-\frac{1}{9^{2}}\right) .
\end{aligned}
$$

Using these scaling formulae for $A_{a}\left(4 \mathrm{~d}^{9} 5 \mathrm{~d} n \mathrm{f}{ }^{2,4} L_{J}\right)$ and $A_{r}\left(4 \mathrm{~d}^{10} 5 \mathrm{~d}^{2} \mathrm{D}_{3 / 2} 4 \mathrm{~d}^{9} 5 \mathrm{~d} n \mathrm{f}^{2,4} L_{J}\right)$, we calculated $Q_{d}\left(4 \mathrm{~d}^{10} 5 \mathrm{~d}-\right.$ $\left.{ }^{2} \mathrm{D}_{3 / 2}-4 \mathrm{~d}^{9} 5 \mathrm{~d} n \mathrm{f}{ }^{2,4} L_{J}\right)$ as a function of $n$ and then, using equation (9) for $E_{S}$, the sums over $n$ for $\alpha_{d}\left(4 \mathrm{~d}^{10}, 4 \mathrm{~d}^{10} 5 \mathrm{~d}^{2} \mathrm{D}_{3 / 2}\right)$ versus $T_{e}$

To estimate $Q_{d}(j, i)$ in equation (3) for autoionizing states $i$ with high $n$ for the $4 \mathrm{~d}^{9} 6 l^{\prime} n l$ states (for example, for the $4 \mathrm{~d}^{10} 6 \mathrm{p}-4 \mathrm{~d}^{9} 6 \mathrm{p} n \mathrm{p}$ dipole transitions), we used the calculated data for $n=7$ and the $1 / n^{3}$ scaling law for $A_{a}$ and $A_{r}$ as was shown above in equations (5)-(9).

The contributions from different DR channels are illustrated in figure 3 where the DR rate coefficient associated with the $4 \mathrm{f}^{2} \mathrm{~F}_{5 / 2}$ and $5 \mathrm{~d}^{2} \mathrm{D}_{3 / 2}$ excited levels is shown as a function of electron temperature. The combined contributions from the autoionizing $4 \mathrm{~d}^{9} 4 \mathrm{f} n l$ and $4 \mathrm{~d}^{9} 5 \ln l^{\prime}$ configurations are represented by curves ' 1 ', ' 2 ', and ' 4 ' for $n=7-9, n=5-9$ and $n=10-1000$, respectively. One can see from these plots that inclusion of the high- $n 4 \mathrm{~d}^{9} 4 \mathrm{f} n l$ and $4 \mathrm{~d}^{9} 5 \ln l^{\prime}$ configurations (cf curve labelled 'scaled') is very important for obtaining the final value of $\alpha_{d}\left(4 \mathrm{~d}^{10}, j\right)$ with $j=4 \mathrm{f}^{2} \mathrm{~F}_{5 / 2}$ and $5 \mathrm{~d}^{2} \mathrm{D}_{3 / 2}$.

Curve ' 3 ' represents the term in equation (4) obtained by summing over the autoionizing $4 \mathrm{~d}^{9} 6 l^{\prime} n l$ configurations with $n=6-7$. Additional contributions were calculated by summing the $4 \mathrm{~d} 6 l^{\prime} n l$ autoionizing configurations from $n=8$ up to $n=1000$. We do not display the resulting curve in the two panels of figure 3 since the corresponding values are too small. The contribution of the $4 \mathrm{~d} 6 \ln l^{\prime}$ autoionizing configurations to the DR rate coefficients $\alpha_{d}\left(4 \mathrm{~d}^{10}, j\right)$ for $j=5 \mathrm{~d}^{2} \mathrm{D}_{3 / 2}$ and $j=4 \mathrm{f}^{2} \mathrm{~F}_{5 / 2}$ is smaller than that for other intermediate states. This is due to the absence of one-electron transitions connecting these states.

The energies $E_{s}$ may strongly affect the $C_{S}^{\mathrm{eff}}(j, i)$ values (see equation (2)). The $E_{s}$ values for the $4 \mathrm{~d}^{9} 6 l^{\prime} 7 l$ autoionizing configurations are larger than the $E_{s}$ values for the $4 \mathrm{~d}^{9} 5 l^{\prime} n l$ and $4 \mathrm{~d}^{9} 4 \mathrm{f} n l$ autoionizing configurations, which leads to a smaller contribution to the $C_{S}^{\text {eff }}(j, i)$ values of the $4 \mathrm{~d}^{9} 6 l^{\prime} 7 l$ configurations, especially for small temperature $T_{e}$ (see figure 3 ). 

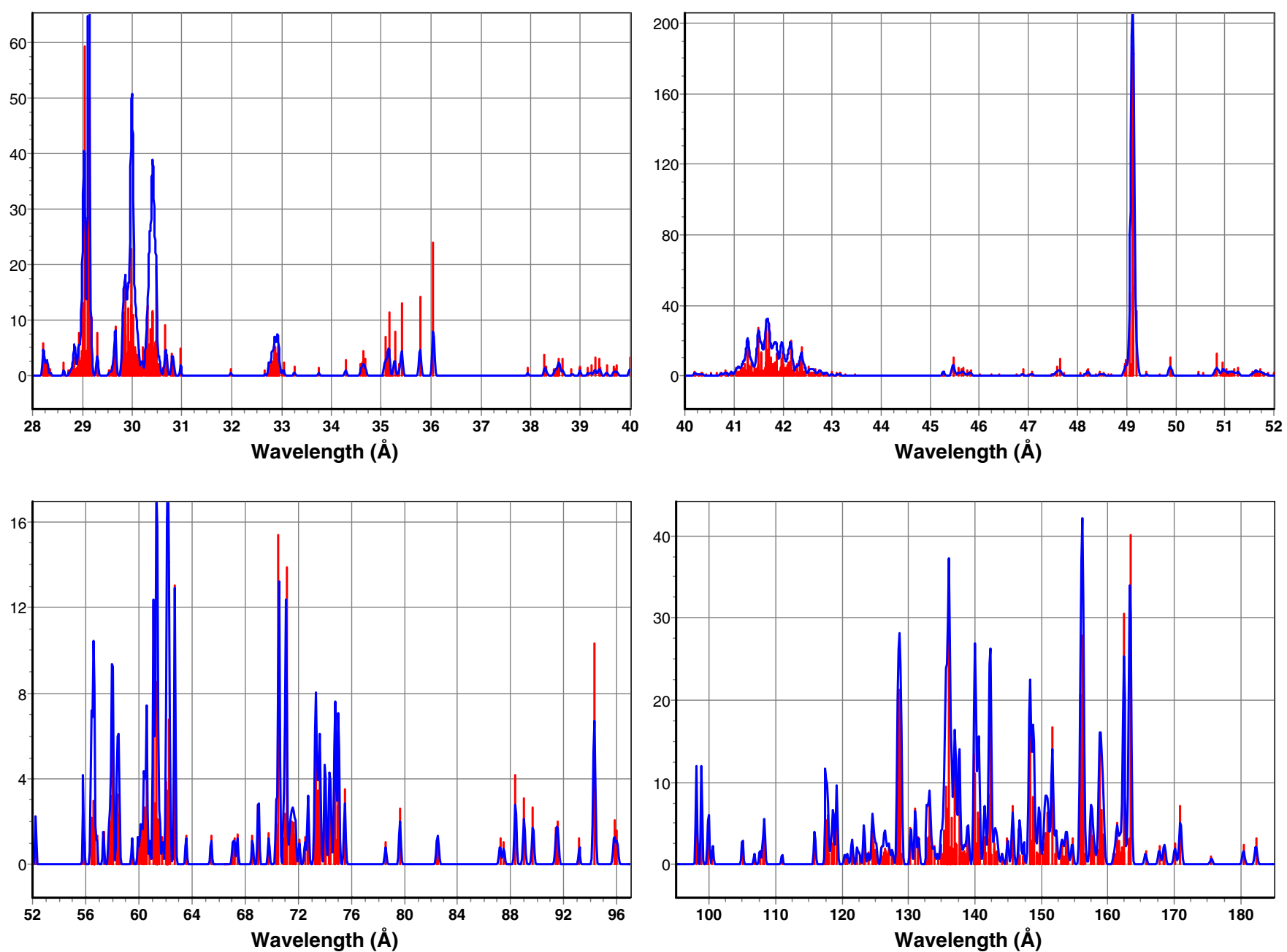

Figure 2. Synthetic spectra (red) of dielectronic satellite lines $\left(\left[4 \mathrm{~d}^{10} n l+4 \mathrm{~d}^{9} 4 \mathrm{f} n l+4 \mathrm{~d}^{9} 5 l 5 l^{\prime}+4 \mathrm{~d}^{9} 5 \mathrm{~s} 6 l\right]-4 \mathrm{~d}^{9} n_{1} l_{1} n_{2} l_{2}\right)$ from the $\mathrm{W}^{27+}$ ion at $\mathrm{T}_{e}=100 \mathrm{eV}$ for $\lambda=28-175 \AA$. A resolving power, $R=\lambda / \Delta \lambda=700$ (top panels), 500 (bottom-left panels) and 400 (bottom-right panel) is assumed to produce a Gaussian profile (blue). The scale in the ordinate is in units of $10^{-14} \mathrm{~cm}^{3} \mathrm{~s}^{-1}$.
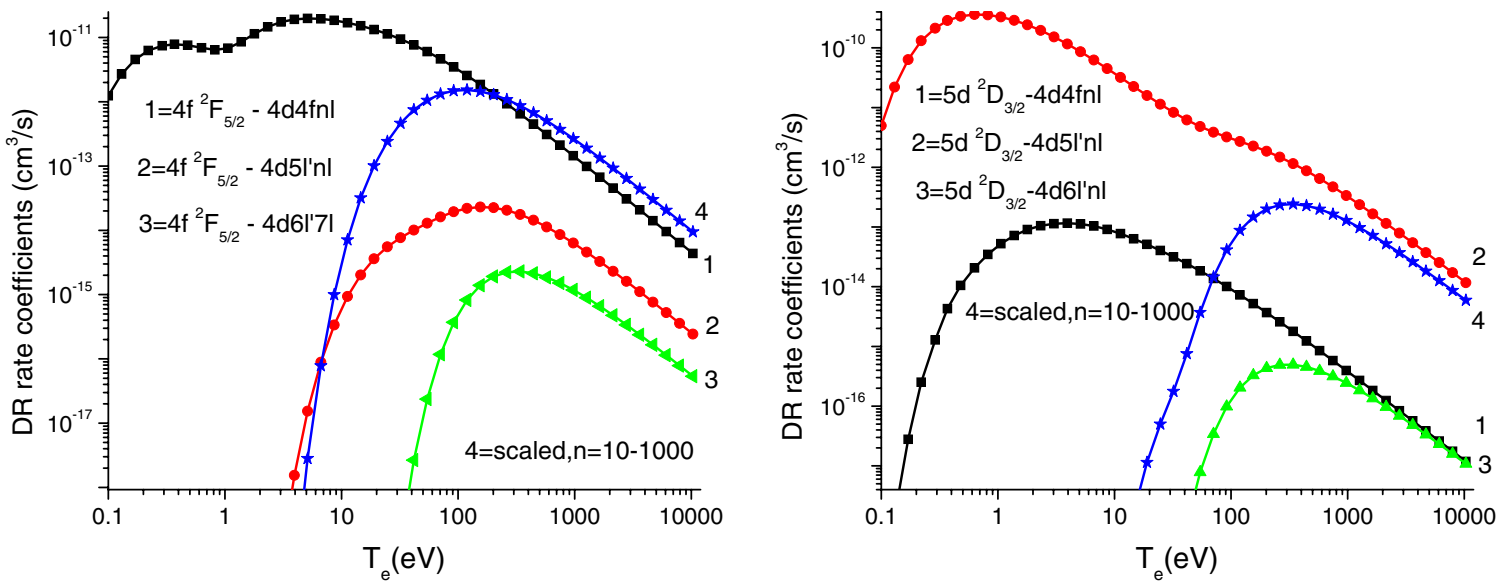

Figure 3. Contribution of the $4 \mathrm{~d}^{9} 4 \mathrm{f} n l(n=7-9), 4 \mathrm{~d}^{9} 5 l^{\prime} n l(n=5-9)$, and $4 \mathrm{~d}^{9} 6 l^{\prime} n l(n=6-7)$ configurations to the DR rate coefficients $\alpha_{d}\left(4 \mathrm{~d}^{10}, j\right)$ for the $j=4 \mathrm{~d}^{10} n l^{2} L_{J}$ states as a function of $T_{e}$ in Ag-like tungsten.

The dependence of the scaled results on the highest value of the principal quantum number $n_{\max }$ was also investigated. We found that there is a small difference for low temperature ( $0.5 \%$ for $T_{e}=10 \mathrm{eV}$ ) with $n_{\max }=20$ as an upper limit instead of $n_{\max }=1000$, and the difference increases with temperatures, reaching $20 \%$ for $T_{e}=100 \mathrm{eV}$.

The calculated values of $\alpha_{d}\left(4 \mathrm{~d}^{10}, j\right)$ as a function of $T_{e}$ in Ag-like tungsten are presented in figure 4. In the four 

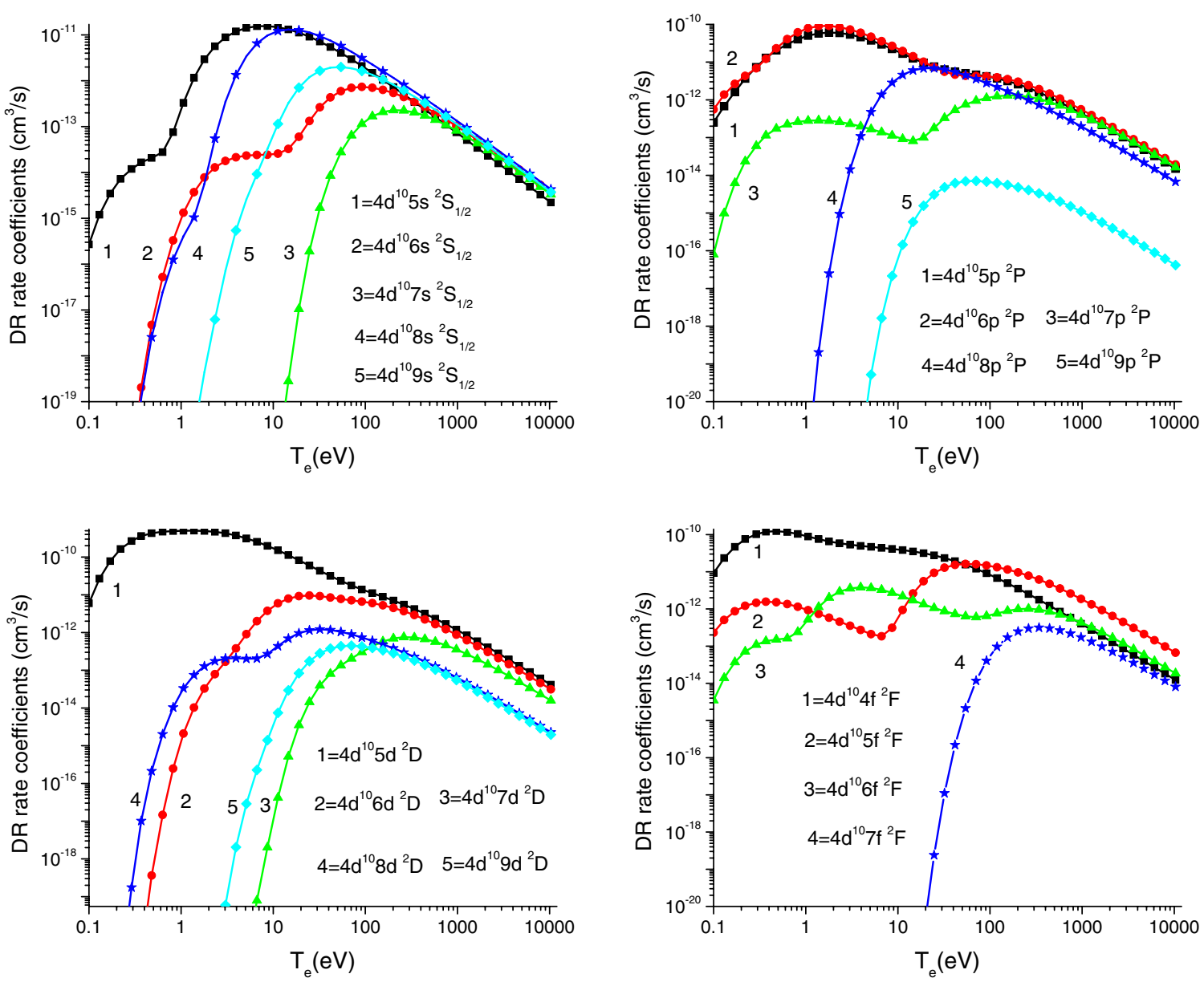

Figure 4. DR rate coefficients $\alpha_{d}\left(4 \mathrm{~d}^{10}, j\right)$ for the $4 \mathrm{~d}^{10} n \mathrm{~s}^{2} \mathrm{~S}_{1 / 2}, 4 \mathrm{~d}^{10} n \mathrm{p}{ }^{2} \mathrm{P}, 4 \mathrm{~d}^{10} n \mathrm{~d}^{2} \mathrm{D}$ and $4 \mathrm{~d}^{10} n \mathrm{f}{ }^{2} \mathrm{~F}$ levels as a function of $T_{e}$ in Ag-like tungsten.

panels, we present the DR rate coefficients $\alpha_{d}\left(4 \mathrm{~d}^{10}, j\right)$ with $j=4 \mathrm{~d}^{10} n \mathrm{~s}^{2} \mathrm{~S}_{1 / 2}, 4 \mathrm{~d}^{10} n \mathrm{p}{ }^{2} \mathrm{P}, 4 \mathrm{~d}^{10} n \mathrm{~d}{ }^{2} \mathrm{D}$ and $4 \mathrm{~d}^{10} n \mathrm{f}{ }^{2} \mathrm{~F}$. The electron temperature for these plots varies from $\mathrm{T}_{e}=0.1$ to $10 \mathrm{keV}$. In order to decrease the number of curves shown, we summed the results for the doublets $\left[{ }^{2} \mathrm{P}_{1 / 2}+{ }^{2} \mathrm{P}_{3 / 2}\right]$, $\left[{ }^{2} \mathrm{D}_{3 / 2}+{ }^{2} \mathrm{D}_{5 / 2}\right]$, and $\left[{ }^{2} \mathrm{~F}_{5 / 2}+{ }^{2} \mathrm{~F}_{7 / 2}\right]$. One can see from figure 4 that the largest values of $\alpha_{d}\left(4 \mathrm{~d}^{10}, j\right)$ with $j=$ $4 \mathrm{~d}^{10} n \mathrm{~s}^{2} \mathrm{~S}_{1 / 2}, 4 \mathrm{~d}^{10} n \mathrm{~d}{ }^{2} \mathrm{D}$, and $4 \mathrm{~d}^{10} n \mathrm{f}^{2} \mathrm{~F}$ are obtained for the smallest value of $n$ in every panel, except in the panel for the $4 \mathrm{~d}^{10} n \mathrm{p}{ }^{2} \mathrm{P}$ excited levels. The $\alpha_{d}\left(4 \mathrm{~d}^{10}, j\right)$ values for the $j=4 \mathrm{~d}^{10} 6 \mathrm{p}{ }^{2} \mathrm{P}$ are a little larger than the $\alpha_{d}\left(4 \mathrm{~d}^{10}, j\right)$ values with $j=4 \mathrm{~d}^{10} 5 \mathrm{p}{ }^{2} \mathrm{P}$. It should be noted that increasing $n$ in $j=4 \mathrm{~d}^{10} n l^{2} L$ leads to the shift of a given curve's maximum towards larger electron temperature: $T_{e}=1 \mathrm{eV}$ for $n=4-6$, up $T_{e}=200 \mathrm{eV}$ for $n=7-9$ (see figure 4 ).

\section{Total dielectronic recombination rate coefficient}

The total DR rate coefficients are obtained by summation of the effective emission rate coefficients $C_{S}^{\text {eff }}(j, i)$ (equation (2)) over all possible intermediate and final singly and doubly excited states:

$$
\alpha_{d}\left(i_{0}\right)=\sum_{i} \sum_{j} C_{S}^{\mathrm{eff}}(j, i)
$$

We have already discussed the contribution from doubly excited states with high- $n$ levels to the DR rate coefficients (sum over $i$ in equation (10)). For the total DR rate coefficient one has to consider the contribution from singly excited states and non-autoionizing doubly excited states (sum over $j$ in equation (10)). There are about 2000 non-autoionizing doubly excited states in the case of Ag-like tungsten. To illustrate the contribution of the $4 \mathrm{~d}^{9} n^{\prime} l^{\prime} n l\left(L_{12} S_{12}\right) L S J$ nonautoionizing states to the total DR rate coefficient, we sum those contributions over all angular moments $\left(L_{12}, S_{12}, L, S\right.$, $J)$. Finally, we find $\alpha_{d}\left(4 \mathrm{~d}^{10}, j\right)$ for the $4 \mathrm{~d}^{9} n^{\prime} l^{\prime} n l$ states.

The sum of the $\alpha_{d}\left(4 \mathrm{~d}^{10}, j\right)$ coefficient over $j$ given by doubly exited, non-autoionizing levels is shown as curve ' 1 ' in figure 5 . In particular, there are 16 doubly excited, non-autoionizing $4 \mathrm{~d}^{9} 4 \mathrm{f} n l(n=4-8)$ configurations and 16 doubly excited, non-autoionizing $4 \mathrm{~d}^{9} 5 l 5 l^{\prime}, 4 \mathrm{~d}^{9} 5 \mathrm{~s} 6 l$ and $4 \mathrm{~d}^{9} 5 \mathrm{p} 6 \mathrm{p}$ configurations. The complete set of the autoionizing configurations includes the $4 \mathrm{~d}^{9} 4 \mathrm{f} 9 l, 4 \mathrm{~d}^{9} 5 \mathrm{~d} n l^{\prime}(n=6-9)$, $4 \mathrm{~d}^{9} 5 \mathrm{f} n l(n=5-9), 4 \mathrm{~d}^{9} 5 g n l(n=5-9)$ and $4 \mathrm{~d}^{9} 6 \ln l(n=$ 6-7).

The contribution from high- $n$ autoionizing levels to the DR rate coefficients involving doubly excited, nonautoionizing configurations is given by curve ' 2 ' in figure 5. We follow the procedure shown above in equations (5)-(9) for the singly excited levels to evaluate 


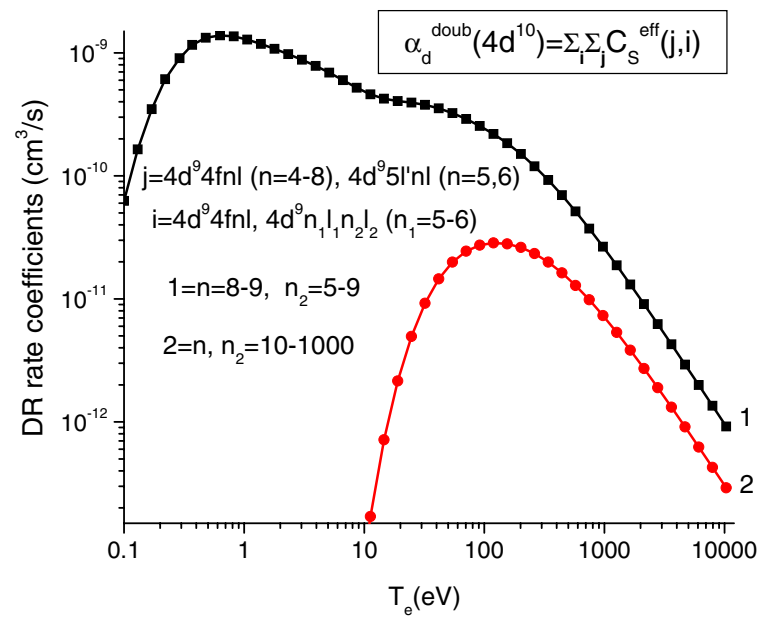

Figure 5. Sum of the contributions from the doubly excited, non-autoionizing $\left(4 \mathrm{~d}^{9} 4 \mathrm{f} n l(n=4-8), 4 \mathrm{~d}^{9} 5 l 5 l^{\prime}, 4 \mathrm{~d}^{9} 5 \mathrm{~s} 6 l\right.$ and $\left.4 \mathrm{~d}^{9} 5 \mathrm{p} 6 \mathrm{p}\right)$ configurations to the total DR rate coefficients $\alpha_{d}\left(4 \mathrm{~d}^{10}\right)$ as a function of $T_{e}$ in Ag-like tungsten.

the scaled values given by curve ' 2 '. In the case of the $4 \mathrm{~d}^{10} n^{\prime} l^{\prime}-4 \mathrm{~d}^{9} n^{\prime} l^{\prime} n l$ transitions, we use empirical scaling laws, which can only be implemented to include one-electron $4 \mathrm{~d}-n \mathrm{p}$, and $4 \mathrm{~d}-n \mathrm{f}$ dipole transitions. That means that we consider the following transitions: $4 \mathrm{~d}^{10} n^{\prime} l^{\prime}-4 \mathrm{~d}^{9} n^{\prime} l^{\prime} n \mathrm{p}$ and $4 \mathrm{~d}^{10} n^{\prime} l^{\prime}-4 \mathrm{~d}^{9} n^{\prime} l^{\prime} n \mathrm{f}$ in the scaling procedure. In the case of the $4 \mathrm{~d}^{9} n_{1} l_{1} n_{2} l_{2}-4 \mathrm{~d}^{9} n_{3} l_{3} n_{4} l_{4}$ transitions, we need to consider the $4 \mathrm{~d}^{9} n_{1} l_{1} n_{2} l_{2}-4 \mathrm{~d}^{9} n_{1} l_{1} n l$ transitions in the scaling procedure. In the case of the $4 \mathrm{~d}^{9} 5 \mathrm{p}^{2}-4 \mathrm{~d}^{9} n_{3} l_{3} n_{4} l_{4}$ transitions, the $4 d^{9} 5 p^{2}-4 d^{9} 5 p n s$ and $4 d^{9} 5 p^{2}-4 d^{9} 5 p n d$ transitions need to be included in the scaling procedure. The final result of the scaling procedure given by curve ' 2 ' of figure 5 shows a maximum at $120 \mathrm{eV}$. Even the maximum value of curve ' 2 ' is less than any value given by curve ' 1 ' of figure 5 . It should be noted that the ratio of the number of transitions from singly excited $4 \mathrm{~d}^{10} n l$ states to autoionizing states to the number of transitions from doubly excited non-autoionizing states to autoionizing states is very small, about $3 \%$ only.

The sum of the contributions from the singly excited $4 \mathrm{~d}^{10} n l$ states to the total DR rate coefficients $\alpha_{d}\left(4 \mathrm{~d}^{10}\right)$ as a function of $T_{e}$ in Ag-like tungsten is shown in figure 6. Curve ' 1 ' displays the $\alpha_{d}^{\text {sing }}\left(4 \mathrm{~d}^{10}\right)=\sum_{i} \sum_{j} C_{S}^{\text {eff }}(j, i)$ coefficient for $j=4 \mathrm{~d}^{10} n l(n=4-9)$ and $i=4 \mathrm{~d}^{9} 4 \mathrm{f} 9 l, 4 \mathrm{~d}^{9} 5 \mathrm{~d} n l(n=6-9)$, $4 \mathrm{~d}^{9} 5 \mathrm{f} n l(n=5-9), 4 \mathrm{~d}^{9} 5 \mathrm{~g} n l(n=5-9)$, and $4 \mathrm{~d}^{9} 6 l^{\prime} n l(n=6-$ 7). Curve ' 2 ' displays the $\alpha_{d}^{\text {sing-sc1 }}\left(4 \mathrm{~d}^{10}\right)=\sum_{i} \sum_{j} C_{S}^{\text {eff }}(j, i)$ coefficient for $j=4 \mathrm{~d}^{10} n l(n=4-9)$ and $i=4 \mathrm{~d}^{9} 4 \mathrm{f} n l$, $4 \mathrm{~d}^{9} 5 \mathrm{~d} n l, 4 \mathrm{~d}^{9} 5 \mathrm{f} n l, 4 \mathrm{~d}^{9} 5 \mathrm{~g} n l(n=10-1000)$ and $4 \mathrm{~d}^{9} 6 \ln l(n=$ $8-1000)$. The details of the scaling procedure were described by equations (5)-(9). Curve ' 3 ' depicts the contributions from singly excited levels with high $n$, i.e. the $4 \mathrm{~d}^{10} n l$ levels with $n>9$. For these levels, the most important transitions are the $4 \mathrm{~d}^{10} n l-4 \mathrm{~d}^{9} n^{\prime}$ f $n l$ and $4 \mathrm{~d}^{10} n l-4 \mathrm{~d}^{9} n^{\prime}$ p $n l$ transitions.

To estimate $Q_{d}(j, i)$ in equation (3) with $j=4 \mathrm{~d}^{10} n l$ and $i=4 \mathrm{~d}^{9} 4 \mathrm{f} n l$ and $4 \mathrm{~d} 5 l^{\prime} n l$ with $n>9$, we used the calculated data for $n=9$ and applied the $1 / n^{3}$ scaling law for the autoionization probabilities $A_{a}$ and energies $E_{S}$. However, the values of $A_{r}$ for the $4 \mathrm{~d}^{10} n l-4 \mathrm{~d}^{9} 4 \mathrm{f} n l, 4 \mathrm{~d}^{10} n l-4 \mathrm{~d}^{9} 5 \mathrm{f} n l, 4 \mathrm{~d}^{10} n l-4 \mathrm{~d}^{9} 5 \mathrm{p} n l$ and

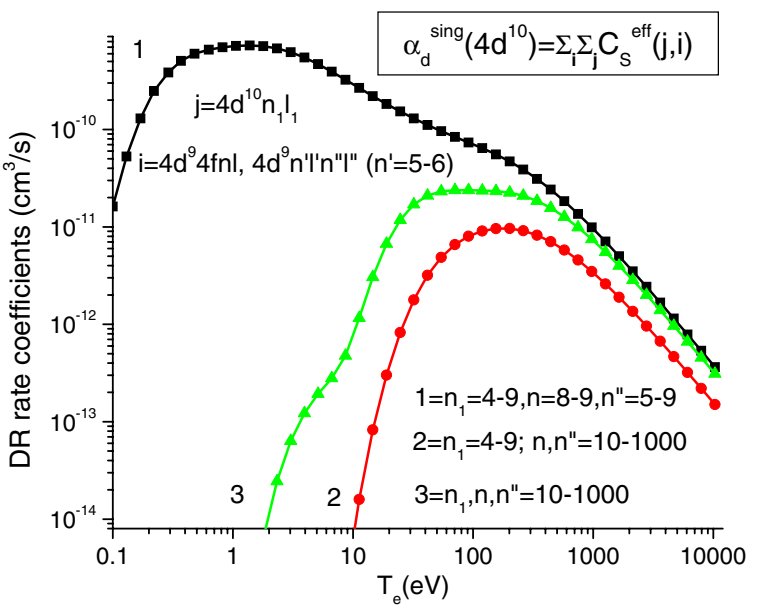

Figure 6. Sum of the contributions from the singly excited $4 \mathrm{~d}^{10} n l$ levels to the total DR rate coefficients $\alpha_{d}\left(4 \mathrm{~d}^{10}\right)$ as a function of $T_{e}$ in Ag-like tungsten.

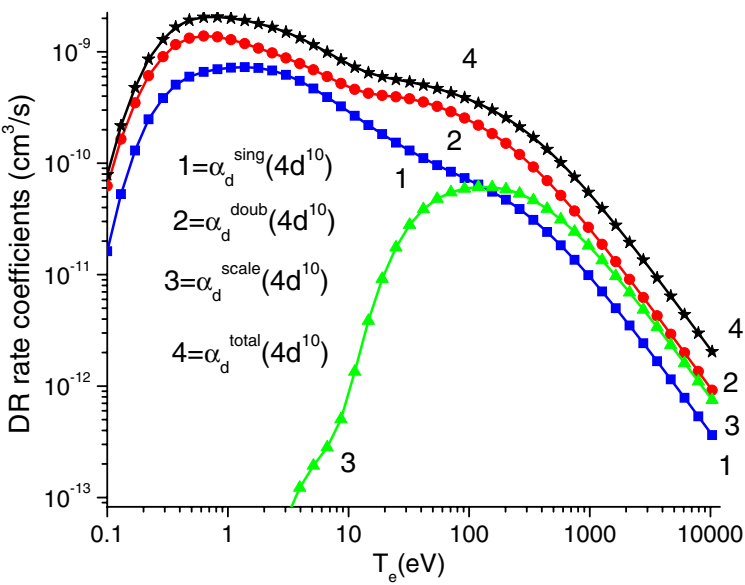

Figure 7. Contributions from the singly excited $\alpha_{d}^{\text {sing }}\left(4 \mathrm{~d}^{10}\right)$ and doubly excited non-autoionizing $\alpha_{d}^{\text {doub }}\left(4 \mathrm{~d}^{10}\right)$ levels to the total DR rate coefficients $\alpha_{d}\left(4 \mathrm{~d}^{10}\right)$ as a function of $T_{e}$ in $\mathrm{Ag}$-like tungsten.

$4 \mathrm{~d}^{10} n l-4 \mathrm{~d}^{9} 6 \mathrm{p} n l$ transitions are almost independent of $n$ since this, in fact, is a one-electron transition where the $n l$ electron is a spectator (see, for example, [46]). Again, the calculated data for $n=7$ and the $1 / n^{3}$ scaling law for $A_{a}$ are used to estimate the intensity factor $Q_{d}(j, i)$ in equation (3) for the $4 \mathrm{~d}^{9} 6 \ln l$ autoionization states $i$ with high $n$. The final value of the $\alpha_{d}^{\text {sing-sc2 }}\left(4 \mathrm{~d}^{10}\right)=\sum_{i} \sum_{j} C_{S}^{\text {eff }}(j, i)$ term for $j=4 \mathrm{~d}^{10} n l$ ( $n=10-1000)$ and $i=4 \mathrm{~d}^{9} 4 \mathrm{f} n l, 4 \mathrm{~d}^{9} 5 \mathrm{~d} n l, 4 \mathrm{~d}^{9} 5 \mathrm{f} n l, 4 \mathrm{~d}^{9} 5 \mathrm{~g} n l$ $(n=10-1000)$, and $4 \mathrm{~d}^{9} 6 \ln l(n=8-1000)$ is presented in curve ' 3 ' of figure 6 . It can be seen from this figure that the $\alpha_{d}^{\text {sing-sc2 }}\left(4 \mathrm{~d}^{10}\right)$ values are larger than the $\alpha_{d}^{\text {sing-sc1 }}\left(4 \mathrm{~d}^{10}\right)$ values by a factor of 10 . The maximum values of curves ' 2 ' and ' 3 ' are near $200 \mathrm{eV}$ and $70 \mathrm{eV}$, respectively. Curve ' 1 ' has a maximum for a very small temperature, i.e. about $1 \mathrm{eV}$.

In figure 7 , we illustrate the results for the total DR rate coefficient $\alpha_{d}\left(4 \mathrm{~d}^{10}\right)$. The electron temperature varies between $0.1 \mathrm{eV}$ and $1 \mathrm{keV}$. The resulting curve labelled ' 4 ' has a maximum at $T_{e} \approx 0.8 \mathrm{eV}$ and very slowly decreases from a maximum value of $2.058 \times 10^{-9} \mathrm{~cm}^{3} \mathrm{~s}^{-1}$ to $2.118 \times$ $10^{-10} \mathrm{~cm}^{3} \mathrm{~s}^{-1}$ at $262 \mathrm{eV}$. Different contributions are shown by 
Table 7. Total DR rate coefficients (in $\mathrm{cm}^{3} \mathrm{~s}^{-1}$ ) $\alpha_{d}^{\text {tot }}=\alpha_{d}^{\text {sing }}+\alpha_{d}^{\text {doub }}$. The singly excited levels are used to evaluate the $\alpha_{d}^{\text {sing }}$ values. The doubly excited non-autoionizing states are used to evaluate the $\alpha_{d}^{\text {doub }}$ values. $\mathrm{A}[\mathrm{B}]$ means $\mathrm{A} \cdot 10^{B}$.

\begin{tabular}{|c|c|c|c|}
\hline $\mathrm{T}_{e}$ & $\alpha_{d}^{\text {sing }}$ & $\alpha_{d}^{\text {doub }}$ & $\alpha_{d}^{\text {total }}$ \\
\hline 0.10 & $1.62[-11]$ & $6.25[-11]$ & $7.87[-11]$ \\
\hline 0.13 & $5.28[-11]$ & $1.64[-10]$ & $2.17[-10]$ \\
\hline 0.17 & $1.30[-10]$ & $3.48[-10]$ & $4.78[-10]$ \\
\hline 0.22 & $2.48[-10]$ & $6.11[-10]$ & $8.59[-10]$ \\
\hline 0.29 & $3.84[-10]$ & $9.04[-10]$ & $1.29[-09]$ \\
\hline 0.37 & $5.07[-10]$ & $1.16[-09]$ & $1.67[-09]$ \\
\hline 0.48 & $5.99[-10]$ & $1.33[-09]$ & $1.92[-09]$ \\
\hline 0.63 & $6.59[-10]$ & $1.39[-09]$ & $2.05[-09]$ \\
\hline 0.82 & $6.97[-10]$ & $1.36[-09]$ & $2.06[-09]$ \\
\hline 1.06 & $7.20[-10]$ & $1.28[-09]$ & $2.00[-09]$ \\
\hline 1.38 & $7.28[-10]$ & $1.18[-09]$ & $1.91[-09]$ \\
\hline 1.79 & $7.16[-10]$ & $1.08[-09]$ & $1.79[-09]$ \\
\hline 2.33 & $6.80[-10]$ & $9.77[-10]$ & $1.66[-09]$ \\
\hline 3.03 & $6.22[-10]$ & $8.79[-10]$ & $1.50[-09]$ \\
\hline 3.94 & $5.48[-10]$ & $7.84[-10]$ & $1.33[-09]$ \\
\hline 5.12 & $4.68[-10]$ & $6.90[-10]$ & $1.16[-09]$ \\
\hline 6.65 & $3.92[-10]$ & $6.00[-10]$ & $9.92[-10]$ \\
\hline 8.65 & $3.25[-10]$ & $5.20[-10]$ & $8.45[-10]$ \\
\hline 11.25 & $2.68[-10]$ & $4.60[-10]$ & $7.28[-10]$ \\
\hline 14.62 & $2.23[-10]$ & $4.25[-10]$ & $6.48[-10]$ \\
\hline 19.00 & $1.90[-10]$ & $4.08[-10]$ & $5.98[-10]$ \\
\hline 24.71 & $1.66[-10]$ & $3.99[-10]$ & $5.65[-10]$ \\
\hline 32.12 & $1.49[-10]$ & $3.88[-10]$ & $5.36[-10]$ \\
\hline 41.75 & $1.35[-10]$ & $3.69[-10]$ & $5.04[-10]$ \\
\hline 54.28 & $1.24[-10]$ & $3.44[-10]$ & $4.68[-10]$ \\
\hline 70.56 & $1.14[-10]$ & $3.14[-10]$ & $4.28[-10]$ \\
\hline 91.73 & $1.05[-10]$ & $2.82[-10]$ & $3.87[-10]$ \\
\hline 119.25 & $9.70[-11]$ & $2.48[-10]$ & $3.45[-10]$ \\
\hline 155.03 & $8.84[-11]$ & $2.12[-10]$ & $3.01[-10]$ \\
\hline 201.54 & $7.91[-11]$ & $1.77[-10]$ & $2.56[-10]$ \\
\hline 262.00 & $6.88[-11]$ & $1.43[-10]$ & $2.12[-10]$ \\
\hline 340.60 & $5.78[-11]$ & $1.12[-10]$ & $1.70[-10]$ \\
\hline 442.78 & $4.69[-11]$ & $8.60[-11]$ & $1.33[-10]$ \\
\hline 575.61 & $3.68[-11]$ & $6.43[-11]$ & $1.01[-10]$ \\
\hline 748.30 & $2.80[-11]$ & $4.71[-11]$ & $7.51[-11]$ \\
\hline 972.78 & $2.09[-11]$ & $3.39[-11]$ & $5.47[-11]$ \\
\hline 1264.62 & $1.52[-11]$ & $2.41[-11]$ & $3.93[-11]$ \\
\hline 1644.01 & $1.09[-11]$ & $1.69[-11]$ & $2.78[-11]$ \\
\hline 2137.21 & $7.70[-12]$ & $1.18[-11]$ & $1.95[-11]$ \\
\hline 2778.37 & $5.39[-12]$ & $8.14[-12]$ & $1.35[-11]$ \\
\hline 3611.88 & $3.74[-12]$ & $5.60[-12]$ & $9.34[-12]$ \\
\hline 4695.44 & $2.58[-12]$ & $3.83[-12]$ & $6.41[-12]$ \\
\hline 6104.07 & $1.77[-12]$ & $2.62[-12]$ & $4.39[-12]$ \\
\hline 7935.30 & $1.21[-12]$ & $1.78[-12]$ & $2.99[-12]$ \\
\hline 10315.89 & $8.25[-13]$ & $1.21[-12]$ & $2.03[-12]$ \\
\hline
\end{tabular}

curves ' 1 ' through ' 3 '. Curve ' 1 ' depicts the $\alpha_{d}^{\text {sing } 1}\left(4 \mathrm{~d}^{10}\right)=$ $\sum_{i} \sum_{j} C_{S}^{\mathrm{eff}}(j, i)$ coefficient for $j=4 \mathrm{~d}^{10} n l(n=4-9)$ and $i=4 \mathrm{~d}^{9} 4 \mathrm{f} 9 l, 4 \mathrm{~d}^{9} 5 \mathrm{~d} n l(n=6-9), 4 \mathrm{~d}^{9} 5 \mathrm{f} n l \quad(n=5-$ 9), 4d $95 \mathrm{~g} n l\left(n=5-9\right.$, and $4 \mathrm{~d}^{9} 6 l^{\prime} n l(n=6-7)$. Curve ' 2 ' displays $\alpha_{d}^{\text {doub1 }}\left(4 \mathrm{~d}^{10}\right)=\sum_{i} \sum_{j} C_{S}^{\mathrm{eff}}(j, i)$ for $j=$ $4 \mathrm{~d}^{9} 4 \mathrm{f} n l(n=4-8), 4 \mathrm{~d}^{9} 5 l 5 l^{\prime}, 4 \mathrm{~d}^{9} 5 \mathrm{~s} 6 l$ and $4 \mathrm{~d}^{9} 5 \mathrm{p} 6 \mathrm{p}$ doubly excited non-autoionizing states and $i=4 \mathrm{~d}^{9} 4 \mathrm{f} 9 l, 4 \mathrm{~d}^{9} 5 \mathrm{~d} n l$ $(n=6-9), 4 \mathrm{~d}^{9} 5 \mathrm{f} n l(n=5-9), 4 \mathrm{~d}^{9} 5 \mathrm{~g} n l(n=5-9)$ and $4 \mathrm{~d}^{9} 6 l^{\prime} n l(n=6-7)$ autoionizing states. Curve ' 3 ' shows the combined scaled contributions illustrated by curve ' 2 ' of figure $5\left(\alpha_{d}^{\text {doub-sc }}\left(4 \mathrm{~d}^{10}\right)\right)$ and curves ' 2 ' and ' 3 ' of figure 6 $\left(\alpha_{d}^{\text {sing-sc1 }}\left(4 \mathrm{~d}^{10}\right)+\alpha_{d}^{\text {sing-sc12 }}\left(4 \mathrm{~d}^{10}\right)\right)$. It is evident from figure 7 that the largest contribution to the total DR rate coefficient $\alpha_{d}\left(4 \mathrm{~d}^{10}\right)$ comes from curve ' 2 ' that describes the contribution from the doubly excited non-autoionizing states. It should be noted that this large contribution from the doubly excited non-autoionizing states was not observed previously for Ag-like ions [46]. The 20 doubly excited non-autoionizing levels were considered in the case of the Ag-like $\mathrm{Xe}^{7+}[46]$; however, the contribution from those levels was negligible for the total DR rate coefficient $\alpha_{d}\left(4 \mathrm{~d}^{10}\right)$.

The partial rate coefficients $\alpha_{d}^{\text {doub }}, \alpha_{d}^{\text {sing }}$, and the total rate coefficient $\alpha_{d}^{\text {tot }}$ are presented in table 7 for $\mathrm{T}_{e}=$ $0.1 \mathrm{eV}-10.315 \mathrm{keV}$ on a logarithmic grid $T_{e}=\left(0.1 \times 1.3^{N-1}\right)$ $\mathrm{eV}$ with $N=1-45$. The coefficient $\alpha_{d}^{\text {doub }}$ is the sum of the contributions $\alpha_{d}^{\text {doub1 }}$ and $\alpha_{d}^{\text {doub-sc }}$ shown as curves '1' and '2' of figure 5, respectively. The coefficient $\alpha_{d}^{\text {sing }}$ is the sum of the contributions $\alpha_{d}^{\text {sing1 }}, \alpha_{d}^{\text {sing-sc1 }}$ and $\alpha_{d}^{\text {sing-sc2 }}$ shown as curves ' 1 ', '2' and ' 3 ' of figure 6 , respectively. The last column of table 7 lists the sum of these two contributions, $\alpha_{d}^{\text {tot }}\left(4 \mathrm{~d}^{10}\right)$. Comparison of the results listed in the columns denoted $\alpha_{d}^{\text {sing }}$ and $\alpha_{d}^{\text {doub }}$ shows that the $\alpha_{d}^{\text {doub }}$ values are larger than the $\alpha_{d}^{\text {sing }}$ values by a factor of $2-3$.

\section{Conclusion}

Accurate atomic data are very important for plasma kinetic modelling, astrophysics, laser spectroscopy and other fields. Our calculations present benchmark data for many yet unmeasured properties of tungsten ions and are of particular importance for the diagnosis of plasma containing tungsten over a broad range of temperatures. In particular, we presented the calculation of a large set of atomic data for dielectronic recombination of the Pd-like $\mathrm{W}^{28+}$ ion into $\mathrm{W}^{27+}$. Energy levels, wavelengths, radiative transition probabilities and autoionization rates were calculated for several thousand levels and millions of transitions in $\mathrm{W}^{27+}$ using three theoretical methods, namely the relativistic many-body perturbation theory method (RMBPT code), the multiconfiguration relativistic Hebrew University Lawrence Atomic Code (HULLAC code) and the Hartree-Fockrelativistic method (COWAN code). The calculated atomic data were used to determine the characteristics of dielectronic satellite lines as well as the DR rate coefficients.

The doubly excited configurations $4 \mathrm{~d}^{9} 4 \mathrm{f} n l,(n=4-9)$, $4 \mathrm{~d}^{9} 5 l^{\prime} n l,(n=5-9)$ and $4 \mathrm{~d}^{9} 6 l^{\prime} n l(n=6-7)$ states were taken into account to derive the DR rate coefficients. The levelselective DR rate coefficients from the ground state of Pdlike $\mathrm{W}$ ion to the singly excited $4 \mathrm{~d}^{10} n l(n=5-9)$ as well as to the doubly excited $4 \mathrm{~d}^{9} 4 \mathrm{f} n l(n=4-7), 4 \mathrm{~d}^{9} 4 \mathrm{f} 8 \mathrm{~s}, 4 \mathrm{~d}^{9} 5 \mathrm{~s} 5 l$, $4 \mathrm{~d}^{9} 5 \mathrm{p} 5 \mathrm{l}, 4 \mathrm{~d}^{9} 5 \mathrm{~d}^{2}, 4 \mathrm{~d}^{9} 5 \mathrm{~s} 6 l$ and $4 \mathrm{~d}^{9} 5 \mathrm{p} 6 \mathrm{~s}$ levels in Ag-like W were calculated. Contributions from the doubly excited levels $4 \mathrm{~d}^{9} 4 \mathrm{f} n l$ and $4 \mathrm{~d}^{9} 5 l^{\prime} n l$ with $n \geqslant 10$ to the DR rate coefficients were estimated by extrapolation of all atomic parameters.

The doubly excited $4 d^{9} 5 p 6 p, 4 d^{9} 5 d 5 f, 4 d^{9} 4 f 7 f, 4 d^{9} 4 f 8 s$, $4 d^{9} 4 \mathrm{f} 8 \mathrm{p}$ and $4 d^{9} 4 \mathrm{f} 8 \mathrm{~d}$ configurations have levels which may be autoionizing or non-autoionizing. Those levels dramatically increase the value of the DR rate coefficients at low temperature. In particular, the contribution to the total DR rate coefficient $\alpha_{d}^{\text {tot }}$ from doubly excited non-autoionizing states $\alpha_{d}^{\text {doub }}$ is larger by a factor of 2-3 than the contribution 
from the singly excited states $\alpha_{d}^{\text {sing }}$. We also showed that the contributions of highly excited levels are important for the calculation of the total DR rate.

In a typical plasma environment, Pd-like tungsten coexists with neighbouring charge states. Additional work will be required to generate the atomic data for those ions, to model the resulting emission, and to accurately determine the ionization balance.

\section{Acknowledgments}

This research was sponsored by DOE under OFES grant DEFG02-08ER54951 and in part under the NNSA Cooperative agreement DE-FC52-06NA27588. Work at the Lawrence Livermore National Laboratory was performed under auspices of the DOE under contract DE-AC52-07NA2344.

\section{References}

[1] Skinner C H 2008 Can. J. Phys. 86285

[2] Reinke M, Beiersdorfer P, Howard N T, Magee E W, Podpaly Y, Rice J E and Terry J L 2010 Rev. Sci. Instrum. $8110 \mathrm{D} 736$

[3] Riccardo V, Firdaouss M, Joffrin E, Matthews G, Mertens P, Thompson V and Villedieu E 2009 Phys. Scr. T 138014033

[4] Rohde V, Balden M, Lunt T and the ASDEX Upgrade Team 2009 Phys. Scr. 2009014024

[5] Isler R C, Neidigh R V and Cowan R D 1977 Phys. Lett. 63A 295

[6] Hinnov E and Mattioli M 1978 Phys. Lett. 66A 109

[7] Hinnov E, Bol K, Dimock D, Hawryluk R J, Johnson D, Mattioli M, Meservey E and von Goeler S 1978 Nucl. Fusion 181305

[8] Asmussen K, Fournier K B, Laming J M, Neu R, Seely J F, Dux R, Engelhardt W, Fuchs J C and Team A U 1998 Nucl. Fusion 38967

[9] Chowdhuri M B, Morita S, Goto M, Nishimura H, Nagai K and Fujioka S 2007 Plasma Fusion Res. 21060

[10] Harte C S, Suzuki C, Kato T, Sakaue H A, Kato D, Sato K, Tamura N, Sudo S, D'Arcy R and Sokell E 2010 J. Phys. B: At. Mol. Opt. Phys. 43205004

[11] Podpaly Y, Rice J E, Beiersdorfer P, Reinke M L, Clementson J and Barnard H 2010 Can. J. Phys. at press

[12] Clementson J, Beiersdorfer P, Roquemore A L, Skinner C H, Manseld D K, Hartzfeld K and Lepson J K 2010 Rev. Sci. Instrum. 81 10E326

[13] Sugar J and Kaufman V 1980 Phys. Rev. A 212096

[14] Safronova U I, Safronova A S and Beiersdorfer P 2009 At. Data Nucl. Data Tables 95751

[15] Safronova U I, Safronova A S and Beiersdorfer P 2009 J. Phys. B: At. Mol. Opt. Phys. 42165010

[16] Biedermann C and Radtke R 2009 AIP Conf. Proc. 116195

[17] Biedermann C, Radtke R, Seidel R and Behar E 2009 J. Phys.: Conf. Ser. 163012034

[18] Meng F-C, Zhou L, Huang M, Chen C-Y, Wang Y-S and Zou Y-M 2009 J. Phys. B: At. Mol. Opt. Phys. 42105203

[19] Meng F-C, Chen C-Y, Wang Y-S and Zou Y-M 2008 J. Quant. Spectrosc. Radiat. Transfer 1092000

[20] Dalhed S, Nilsen J and Hagelstein P 1986 Phys. Rev. A 33264
[21] Behar E, Mandelbaum P and Schwob J L 1999 Phys. Rev. A 592787

[22] Peleg A, Behar E, Mandelbaum P and Schwob J L 1998 Phys. Rev. A 573493

[23] Behar E, Mandelbaum P and Schwob J L 1999 Eur. Phys. J. D 7157

[24] Kramida A E and Shirai T 2009 At. Data Nucl. Data Tables 95305

[25] Safronova U I and Safronova A S 2010 J. Phys. B: At. Mol. Opt. Phys. 43074026

[26] Ivanova E P 2009 At. Data Nucl. Data Tables 95786

[27] Cheng K-T and Kim Y-K 1979 J. Opt. Soc. Am. 69125

[28] Safronova U I and Safronova A S 2009 Can. J. Phys. 8783

[29] Kaufman V and Sugar J 1981 Phys. Scr. 24738

[30] Sugar J and Kaufman V 1981 Phys. Scr. 24742

[31] Kaufman V and Sugar J 1984 J. Opt. Soc. Am. 138

[32] Kaufman V and Sugar J 1985 J. Opt. Soc. Am. 2426

[33] Sugar J, Kaufman V and Rowan W L 1993 J. Opt. Soc. Am. 101321

[34] Churilov S S and Joshi Y N 2002 Phys. Scr. 6540

[35] Saloman E B 2004 J. Phys. Chem. Ref. Data 33765

[36] Safronova U I and Kato T 1998 J. Phys. B: At. Mol. Opt. Phys. 312501

[37] Safronova U I and Kato T 1996 Phys. Scr. 53461

[38] Kato T, Safronova U I and Ohira M 1997 Phys. Scr. 55185

[39] Safronova U I, Kato T and Ohira M 1997 J. Quant. Spectrosc. Radiat. Transfer 58193

[40] Murakami I, Safronova U I, Vasilyev A A and Kato T 2005 At. Data Nucl. Data Tables 901

[41] Murakami I, Safronova U I and Kato T 2002 Can. J. Phys. 801525

[42] Murakami I, Safronova U I and Kato T 1999 J. Phys. B: At. Mol. Opt. Phys. 325351

[43] Safronova U I and Mancini R 2009 At. Data Nucl. Data Tables 9554

[44] Murakami I, Kato T, Kato D, Safronova U I, Cowan T E and Ralchenko Y 2006 J. Phys. B: At. Mol. Opt. Phys. 392917

[45] Murakami I, Safronova U I and Kato T 2009 J. Phys.: Conf. Ser. 163012061

[46] Safronova U I, Bista R, Bruch R and Ralchenko Yu 2009 J. Phys. B: At. Mol. Opt. Phys. 42015001

[47] ftp://aphysics.lanl.gov/pub/cowan

[48] Bar-Shalom A, Klapisch M and Oreg J 2001 J. Quant. Spectrosc. Radiat. Transfer 71169

[49] Safronova M S, Johnson W R and Derevianko A 1999 Phys. Rev. A 604476

[50] Safronova U I, Savukov I M, Safronova M S and Johnson W R 2003 Phys. Rev. A 68062505

[51] Safronova U I, Johnson W R, Safronova M S and Albritton J R 2002 Phys. Rev. A 66042506

[52] Safronova U I, Johnson W R, Safronova M S and Albritton J R 2002 Phys. Rev. A 66052511

[53] http://das101.isan.troitsk.ru/cowan.htm

[54] Safronova U I and Safronova M S 2008 Phys. Rev. A 78052504

[55] Savukov I M and Johnson W R 2000 Phys. Rev. A 62052512

[56] Safronova U I, Johnson W R and Albritton J R 2001 At. Data Nucl. Data Tables $\mathbf{7 7} 215$

[57] Dubau J and Volonte S 1980 Rep. Prog. Phys. 43199

[58] Safronova U I, Bista R, Bruch R and Merabet H 2008 Can. J. Phys. 86131

[59] Safronova U I, Cowan T E and Jonson W R 2005 Can. J. Phys. 83813

[60] Safronova U I, Tolstikhina I Y, Bruch R, Tanaka T, Hao F and Schneider D 1993 Phys. Scr. 47364 